\title{
Mothers' Socialization of Emotions and Socio-Emotional Competences in Children with and without Intellectual Disabilities in Belgium and Quebec
}

\author{
Nathalie Nader-Grosbois ${ }^{1 *}$, Emilie Jacobs ${ }^{1}$, Diane Morin ${ }^{2}$ \\ ${ }^{1}$ Chair Baron Frère in Specialeducation, Psychological Sciences Research Institute, UCLouvain, Louvain-La-Neuve, Belgium \\ ${ }^{2}$ Psychology Department, Université du Québec à Montréal, Montréal, Québec, Canada \\ Email:*nathalie.nader@uclouvain.be
}

How to cite this paper: Nader-Grosbois, N., Jacobs, E., \& Morin, D. (2022). Mothers' Socialization of Emotions and Socio-Emotional Competences in Children with and without Intellectual Disabilities in Belgium and Quebec. Psychology, 13, 135-176.

https://doi.org/10.4236/psych.2022.131010

Received: November 9, 2021

Accepted: January 26, 2022

Published: January 29, 2022

Copyright (อ 2022 by author(s) and ScientificResearch Publishing Inc.

This work is licensed under the

CreativeCommons Attribution

International

License (CC BY 4.0).

http://creativecommons.org/licenses/by/4.0/

\begin{abstract}
This study aims first to compare Belgian and Quebec mothers' emotionrelated reactions toward typically developing (TD) children and children with intellectual disabilities (ID) and examine whether these practices vary depending on individual factors and culture. Second, it investigates to which extent these maternal reactions contribute to Theory of Mind (ToM), emotion regulation and social skills in these children. The participants were 72 children with and without ID matched on developmental age and their mothers from Belgium and Quebec. Mothers completed questionnaires on their reactions to emotions felt by their children's and on the children's socio-emotional abilities. One-way MANCOVAs indicated effects only for ID samples of culture, considering maternal educational level as a covariate, on maternal unsupportive reactions to negative emotions and, specifically, on unsupportive (punition and minimization) and supportive (problem-focused, comforting and encouragement) reactions to negative emotions. Correlational and stepwise regression analyses showed that individual factors, such as developmental age, ID factor in children, mothers' educational level and culture explained a percentage of the variance in mothers' reactions to positive or negative emotions in children. Moreover, individual factors and mothers' reactions to positive or negative emotions in children explained a percentage of the variance in children's ToM abilities, emotion regulation and social skills. Findings highlighted the importance of considering cultural and educational factors as well as individual characteristics to understand how ToM, emotion regulation and social adjustment in children with and without ID are influenced by these factors in addition to parents' reactions to emotions. It provides useful information for assisting parents in supporting their child's socio-emotional abilities.
\end{abstract}




\section{Keywords}

Parental Socialization of Emotions, Culture, Intellectual Disabilities

\section{Introduction}

The present article aims to question how socio-emotional abilities in children with intellectual disabilities (ID) and typically developing (TD) children are influenced by individual, cultural factors and parents' practices of emotion socialization. In comparing two French-speaking countries (Quebec and Belgium), the interest of this exploratory study is based on the fact that both cultural groups speak French (although there is some variability in the use of words and expressions), promote similar educational values, oriented by autonomy and social inclusion of preschoolers at risk and children with ID, and are working to improve the assistance given to parents to support their children's social skills and adjustment. Despite these partial cross-cultural similarities, other aspects of each culture could cause the ERSBs in these mothers to vary. Among aspects that could differ between Quebec and Belgium, there are notable differences in schools' programs, in the implementation of evidence-based programs focused on socioemotional abilities, in parent-professional partnership during accompaniment and in teachers' education. In order to base this study, reference should be made to relevant theories and the state of the art about skills in theory of mind, emotion regulation and social adjustment of TD children and with ID and about parents' practices of socialization of emotions, including their reactions to children's emotions.

Theory of mind, emotion regulation, social adjustment and individual factors in children

In a family setting and in all social environments, including school, children with and without developmental disorders are confronted, in their interactions with peers and adults, with emotional situations inducing joy, sadness, fear, anger and even guilt, shame, surprise...in them or in their partners (Denham et al., 2003, Saarni, 1999). They must be able to successfully identify emotions through facial or postural expressions in other people, their voice intonation and the emotional terms they use, and to respond adequately. They could share positive or negative emotions in certain circumstances, for example in showing empathic preoccupation and giving comfort when encountering fear or distress in others, or in enjoying pleasant situations with others (Hoffman, 2000). The children are likely to infer mental states in others and differentiate their own mental states (Theory of mind, ToM (Flavell, 1999)) in order to regulate their emotions, adjust their behavior in accordance with social norms or rules, and cooperate with peers and adults in play or learning situations or in daily life events (Abbeduto \& Murphy, 2004; Astington \& Baird, 2005; Astington \& Edward, 2010; Baurain \& Nader-Grosbois, 2012, 2013a, 2013b; Nader-Grosbois, 2011; Yeates et al., 2007). The more children are able to mobilize their socio-emotional competences in 
their life experience, notably in a family setting and in school, the more they experience positive social relationships and ensure their well-being. Therefore, three main domains should be developed and supported: 1) Social cognition including theory of mind (understanding of affective states, emotions and desires, or of cognitive mental states such as beliefs, intentions, thoughts, pretense...) and social information processing; 2) Emotion regulation during social interactions; 3) Social adjustment in relationship with peers and adults (Deneault \& Ricard, 2013; Nader-Grosbois, 2011; Yeates et al., 2007).

However, several risk and protection factors could play a role in the development of these socio-emotional domains (Denham, 1998; Denham \& Kochanoff, 2002; Denham \& Weissberg, 2004). These factors include individual intra-personal characteristics in children, their chronological and developmental ages, the presence or absence of disability or disorder, their temperament or personality, their cognitive abilities, their communicative or language levels, etc. (Abbeduto \& Murphy, 2004; Astington, Repacholi, \& Slaughter, 2003). The environmental factors relate to peers, parents, family, and educational environments. For example, in preschool and school, socio-emotional activities conceived in evidencebased programs, implemented in the classroom, and interactions with teachers, and between students, could also help to support this development (Howes, 2000; Honoré, 2020). About specifically parents' factors and their practices of socialization of emotions, some models explained how parents contribute to their child's development in theory of mind, in emotion regulation and their social adjustment.

\section{Parental factors and models explaining links between socio-emotional} abilities and parents' socialization of emotions

In order to explain how parents could help or hinder the development of socio-emotional competences (including ToM, emotion regulation and social adjustment) and well-being in children, authors conceived theoretical models that guided empirical studies on links between parents' emotion socialization and various emotional and social skills in children. Empirical studies were often founded on "Emotion-coaching philosophy" (Gottman, Katz, \& Hooven, 1996), or the "Heuristic model of the socialization of emotion" (Eisenberg, Cumberland \& Spinrad, 1998a) or the "Family Socialization of Emotion Regulation" (Morris et al., 2007), or the conceptual framework of Denham and collaborators (Denham, 1998; Denham et al., 1994, 1997, 2004, 2007; Denham \& Kochanoff, 2002). The present study refers to the model of Eisenberg et al. (1998a, 1998b). This model focuses on parental emotion-related socialization behaviors (ERSBs), including reactions by mothers and fathers toward their child's emotions, conversations about emotions, emotional expressions, in relation to distinct socio-emotional abilities in children. Eisenberg et al. (1998a, 1998b) consider individual intra-personal factors in children and parents. They distinguish two main categories of parental reactions, "supportive" vs "unsupportive", related to positive or negative emotions felt by children (Eisenberg et al., 1998a, 
1998b; Eisenberg et al., 1996; Fabes et al., 2002). The "supportive" parental reactions to children's positive emotions correspond to "encouragement" to express their emotions and "socialization", while the "unsupportive" parental reactions are "reprimand" and "discomfort". When the children express negative emotions, parents could show either "supportive" reactions such as "encouragement" of emotional expression, "comforting", "problem-focused responses", or, on the contrary, "unsupportive reactions", by displaying "distress" or "punition" or "minimizing" the child's emotions. These distinct reactions have been considered in numerous empirical studies, by using a mainly self-report questionnaire and sometimes, observational design of dyads parent-child.

As reported by previous studies, several parental factors could influence children's emotional expression, emotion regulation, theory of mind and social competences. Parents' factors include intra-personal factors, gender, educational, socio-cultural, economic levels, personality, styles (warm, responsive, overprotective, controlling, authoritarian), their emotional openness or competences and their emotion-related beliefs; and as inter-personal factors, their practices of emotion socialization through emotion-related reactions or conversations, e.g. (Denham, Bassett, \& Wyatt, 2007; Eisenberg et al., 2003; Garner \& Estep, 2001; Garner, Jones, \& Miner, 1994; Garner, Robertson, \& Smith, 1997b; Garner \& Spears, 2000; Garner, Dunsmore, \& Southam-Gerrow, 2008; Mazzone \& NaderGrosbois, 2016, 2017a, 2017b, 2017c, 2017d; Jacobs et al., 2019a, 2019b; Wong, Diener, \& Isabella, 2008; Wong, McElwain, \& Halberstadt, 2009). Some studies investigated potential differences in parents' reactions toward TD children and atypically developing children (with externalizing behavior, with autism spectrum disorders or with ID.

\section{About parental reactions}

Concerning parents' reactions to emotions felt by their child, comparative studies have emphasized either similarity or some differences between parents of children with and without ID, at preschool or school age, and matched on their chronological or developmental age. Most studies considered a dichotomic approach in differentiating supportive vs unsupportive reactions, by using a mainly self-report questionnaire. The Coping with Children's Negative Emotions Scale (CCNES (Coutu et al., 2002)) was used by notably Paczkowski \& Baker (2007), Rodas, Zeedyk, \& Baker (2016) and Rodas, Chavira, \& Baker (2017). The Parental Reactions to Positive and Negative Emotions Questionnaire (Daffe \& NaderGrosbois, 2009) was used by Légaré et al. (2019) and Jacobs et al. (2019a, 2019b), in order to compare parents' supportive vs unsupportive reactions to their child's positive and negative emotions, as well as to investigate specific subcategories of reactions. Only one study observed unsupportive reactions in parents of ID children through a self-report questionnaire and a naturalistic home observation (Rodas et al., 2016). In their study, Rodas et al. (2016) observed different results depending on the measure. In fact, when comparing reactions based on the observational measure, mothers displayed more unsupportive reactions than fa- 
thers, while regarding self-report data, mothers reported more supportive reactions compared to fathers, toward their ID children.

Concerning studies using a self-report questionnaire, main results are the following. In a sample of 225 American families, Paczkowski \& Baker (2007) did not find any difference between 97 mothers of children with developmental delay and 128 mothers of TD children who were 4 years old, in terms of their supportive and unsupportive reactions to negative emotions. Légaré et al. (2019) did not find differences in supportive and unsupportive reactions to positive and negative emotions felt by the child, between 40 mothers of TD children and 34 mothers of children with ID from Quebec, when controlling for their developmental age. They suggested that mothers with children with ID have received guidance that could help them to be aware of the positive impact of their support on socio-emotional abilities in their children. Even if other studies indicated that parents of children with ID reported more unsupportive reactions than those of TD children, matched on developmental age (Jacobs et al., 2019a; Rodas et al., 2016; Rodas et al., 2017), they emphasized more nuanced results. Indeed, they revealed differentiated reactions by mothers and fathers of children with ID (Jacobs et al., 2019a; Rodas, et al., 2016, 2017). More precisely, in the study led by Jacobs et al. (2019a) in Belgium, 27 mothers of children with ID displayed more distress and used more punitive and minimizing reactions when their child felt negative emotions, but also more supportive comforting reactions toward their children, than the 27 mothers of TD children. Moreover, mothers of children with ID reacted with more discomfort to positive emotions than those of TD children. However, global supportive reactions to negative emotions and positive emotions, and unsupportive reactions to positive emotions, did not differ between the two groups of mothers. 17 fathers of children with ID displayed more supportive and unsupportive reactions to negative emotions, and more specifically, reported more comforting and problem-focused reactions, and encouragement of emotional expression, to support their child, but more distress, than the 17 fathers of TD children (Jacobs et al., 2019a). When faced with positive emotions in the children with ID, their fathers reacted with more discomfort than those of TD children. To deepen links between parents' reactions and socio-emotional abilities in children, studies have been led at different periods of development.

About links between parental reactions to emotions and ToM, emotion regulation and social adjustment in TD children and children with ID

Most studies investigated the links between parents' reactions and these socio-emotional abilities in TD children at preschool and around school starting age, and children exhibiting externalizing behavior; however, far fewer studies targeted children with ID. Some studies have examined supportive vs unsupportive reactions in mothers and rarely in fathers or in both parents, in relation to ToM, emotion regulation, or social adjustment (in TD children and children with ID or developmental delay (Légaré et al., 2019; Paczkowski \& Baker, 2007; Rodas et al., 2016, 2017)). However, rare studies explored specific reactions in these two categories (in parents of TD children and/or of children with ID (Jacobs 
et al., 2019a, 2019b); in parents of TD and children with autism spectrum disorders (Mazzone \& Nader-Grosbois, 2015, 2016, 2017a, 2017b, 2017c, 2017d)).

As regards parents' reactions and ToM abilities, Jacobs et al. (2019b) examined how individual factors and mothers' and fathers' reactions to emotions predicted ToM in 37 Belgian children with ID. They found that children's chronological age (CA) explained variability in global ToM skills as perceived by mothers, and contribute more specifically to the variability of ToM thoughts (in combination with their global developmental age, GDA) and ToM beliefs, in combination with mothers' problem-focused, minimization, and distress reactions to negative emotions. Fathers' perceptions of their children's ToM skills and specifically about socio-emotional ToM were explained by CA, the ToM beliefs by GDA, and the ToM thoughts by GDA in combination with fathers' comforting and punitive reactions to negative emotions. Interesting results were obtained.

Some studies showed links between negative parenting style and unsupportive parental reactions to children's negative emotions and emotion regulation in TD children at risk of externalizing or internalizing behavior disorders (Eisenberg et al., 2001a, 2001b; Hudson \& Rapee, 2001; Rodas et al., 2016, 2017), including anxiety disorders (Degnan, Almas, \& Fox, 2010; Hudson \& Rapee, 2001; Hurrell, Hudson, \& Schniering, 2015). Rare studies explored this link in dyads comprising children with ID and their parents. Rodas et al. (2016) suggested that parents whose children with ID display difficulties in emotion regulation might exhibit less supportive reactions. Jacobs et al. (2019b) found that emotion regulation in children with ID was predicted by mothers' reprimanding reactions to positive emotions, although the children's CA or GDA better predicted their emotion dysregulation, and also partially varied according to the mothers' educational level.

Other studies showed that parental ERSBs affect children's social adjustment, particularly at preschool age. Supportive parental reactions to children's negative emotions were linked to better social adjustment in children, and inversely, unsupportive parental reactions were associated with poorer social skills in children (Eisenberg et al., 1998a, 1998b; Eisenberg, Fabes, \& Murphy, 1996; Fabes et al., 2001, 2002; Jones et al., 2002) and with greater risk of externalizing or/and internalizing behavior problems, including anxiety disorder in childhood until adolescence, e.g. (Dodge \& Pettit, 2003; Rodas, Zeedyck, \& Baker, 2016). In their study, Paczkowski \& Baker (2007) emphasized the link between unsupportive reactions to negative emotions, and child behavior problems, in children with and without developmental delay. For the entire sample and specifically in the group with delay, the association between mothers' perceptions of self-mastery of life events and child behavior problems in the Child Behavior Checklist (CBCL) was partially mediated by unsupportive reactions. The developmental delay in children moderated the relationship between unsupportive reactions and child behavior problems, and particularly internalizing problems. When mothers displayed low levels of unsupportive reactions, children in the delayed and TD groups had similar levels of problem behaviors. However, when mothers 
showed medium or high levels of non-supportive reactions, children with developmental delay had much higher levels of problem behaviors than TD children. These authors pointed out that parents' ERSBs could have a greater impact on social behaviors in children at developmental risk. Rodas et al. (2016) investigated, in 156 American mother and father dyads and their children with and without ID, when they were 4 to 5 years old, relationships between parents' selfreported unsupportive, negative parenting, parental depression, and children's internalizing behavior problems. The results revealed differences in unsupportive, negative parenting depending on the presence of ID or not, and parent gender. Depression in fathers significantly moderated the relationship between unsupportive parenting and children's internalizing behavior problems. Jacobs et al. (2019a) explored to what extent individual factors and mothers' and fathers' reactions to emotions predict social adjustment or maladjustment in 27 TD children and 27 children with ID from Belgium. Correlation and regression analyses highlighted the fact that children's GDA was a good predictor of variability in social adjustment, particularly the dependent-autonomous dimension, and a low level of internalizing problems. In addition, the combination of GDA and specific reactions (supportive or unsupportive) in mothers or fathers helped to explain variability in some socio-affective dimensions. More specifically, social skills relating to rules, and the aggressive-controlled and egoistical-prosocial dimensions, were better explained by GDA and distress on the part of mothers in response to negative emotions. The anxious-confident dimension was better explained by GDA and maternal problem-focused reactions to negative emotions. The CA and maternal reprimanding reactions to positive emotions contribute to the variability of the resistant-cooperative dimension. The GDA, and also the combination of GDA and specific paternal reactions, including comforting the child when he or she felt negative emotions, predicted social adjustment, notably more controlled social behavior, and reprimanding reactions to positive emotions predicted less depressive expression. These studies showed the relevance of examining not only supportive vs unsupportive reactions, but also each specific reaction in parents in relation to ToM, emotion regulation and different aspects of social adjustment and skills in atypically developing children. Even these findings about links between parents' reactions to emotions and these socioemotional abilities, in considering individual factors in children are very interesting; they did not give information about how cultural and educational factors intervene in these parental practices.

Cultural and educational factors, parents socialization of emotion and socio-emotional abilities in children

Moreover, another factor influences the children's knowledge of social rules, their understanding of how to react and respond adequately in socio-emotional situations and these parental practices of emotion socialization, which corresponds to cultural aspects at different levels (Denham et al., 2004). In line with socio-constructivist conception developed by Vygotsky (1978), during interac- 
tions in a family setting or in social membership groups, the conversations (for example, by using terms relating to emotions, mental states, social experiments, and cultural landmarks) and opportunities of imitation of behaviors help the children to understand mental states, to talk about emotions and to learn how to regulate their emotions and social behavior in reference to social conventions or rules, but in a variable way depending on the culture, values, education and economic levels of families (Eisenberg et al., 1998a, 1998b; Fivush \& Wang, 2005; Garner, 2006; Grusec, 2002; Halberstadt \& Lozada, 2011; Jaramillo et al., 2017; Wang, 2001).

Regarding parents' education level and culture, studies emphasized that these factors influence various components of "parentality". For example, as explained by Parent et al. (2008) and Roskam et al. (2015: p. 175-177), research showed that parents with a low level of education have less cognitive flexibility to adjust their educative behavior or to react appropriately toward their children according to the specificities of situations. They present less competence in taking decisions about which adapted attitude they should use to respond to their child's needs, and their educative values are more oriented to obedience than autonomy in children, which affects their educative behaviors, their cognition of children's abilities, and their self-efficacy beliefs. These components of parentality induce variability in goals and strategies of socialization toward children. Moreover, the importance is given to socio-emotional development, goals and practices of socialization, and the perception of support needs in children vary according to family culture (Claes, Ziba-Tanguay, \& Benoit, 2008; Roskam et al., 2015: p. 188-191).

The main studies were conducted in middle-class families, or some of them specifically in low-income families, e.g. (Garner et al., 1994, 1997a, 1997b; Garner \& Spears, 2000) and in diverse countries and ethnicities (European, American, Asian, African, Indian); however, they rarely explored cross-cultural comparison and parents' differing educational levels to investigate ERSBs and their contribution to socio-emotional development in children. So, their findings about the respective impact of ERSBs during parent-child interactions and children's socio-emotional abilities are embedded in a broader socio-cultural context and beliefs and they may differ in quality, function, and meaning according to different cultures, see (Bornstein \& Bradley, 2003; Dunsmore \& Halberstadt, 1997, 2009; Grusec, 2002; Halberstadt, Denham, \& Dunsmore, 2001; Halberstadt \& Lozada, 2011; Trommsdorff \& Kornadt, 2003). As explained by these authors, depending on variations in cultures, several frames contribute to inducing variability in parents' socialization practices, in general and in emotional situations, as well as variability in children's socio-emotional competences through emotional experience, expression, regulation and understanding. They discussed how these processes could be influenced by relevant cultural frames, including: "collectivist vs individualist" cultures, the "power of distance" in social relationships, the children's place in family and in culture (value of children, relationship with 
children), beliefs about the ways children learn (when, whether, how children learn?) and the value of emotional experience and expression. According to Gottman et al. (1996), social norms, beliefs and education practices vary depending on culture. More concretely, the socio-cultural environment in which the child lives indirectly influences parents' beliefs and socialization goals for their child (Halberstadt \& Lozada, 2011). The parent-child interactions are steeped in the socio-cultural context that "colors" shared activity and shared meaning, by cultural practices, beliefs and interpretations (Keller, 2007). The children incorporate information and internalize values through these experiences, provided by culture, that help them to learn and to display behaviors influencing, in turn, their parents' ERSBs (Keller, 2007; Kuczynski, Marshall, \& Schell, 1997; Vygotsky, 1978).

Cultural models involve beliefs as well as social practices that guide what is considered moral, imperative, and desirable in the domains of emotions and social relationships (Hofstede, 2001). Mesquita \& Walker (2003) discussed differences in culturally functional emotional lives and emphasized distinct aspects that vary across cultures in terms of emotional practices, summarized as follows. Each culture promotes antecedent events or contexts that could imply positive emotions and types of interpretations of events that affect emotion elicitation. The culture may influence which aspects of events are the focus of attention leading to either negative or positive outcomes. The social rules in a cultural model could facilitate or curb the expression of more positive or negative feelings (notably stressful social rules inducing anxiety, as explained by (Hofstede, 2001) and the receptivity to emotions as pleasant or unpleasant experiences. Cultural models also foster culture-specific appraisals reflected in culturally distinct patterns of emotional experience, and the role of personal agency in the control over the emotional events. So, cultural differences could be reflected in emotional expressions and behaviors, and their regulation. Both socio-emotional competences in typically and atypically developing children and parents' socialization of emotions could vary depending on these cultural aspects, notably in Quebec and in Belgium.

To guide research, some models integrated the culture to explain direct and indirect impact on parents' ERSBs, which were developed by Eisenberg et al. (1998a, 1998b), Dunsmore \& Halberstadt (1997, 2009), or Cole \& Tan (2007). In addition, it is relevant to adopt an approach, in line with Kuczynski et al. (1997), for example, of "bidirectional" links between ERSBs in parent-child interactions and socio-emotional competences in children, considering both parents' and child's mutual influence and agency in diverse aspects (Trommsdorff \& Kornadt, 2003).

The variability of parental reactions to children's emotions depending on cultural factors, was rarely examined, particularly in French-speaking countries. Some comparative cross-cultural studies have been conducted on parental emotion expressiveness (Camras, Kolmodin, \& Chen, 2008) or parents' reactions to 
TD children's emotions (Brown et al., 2015). Camras et al. (2008) compared Mainland Chinese, Chinese American and European American mothers' emotional expression toward 115 children who were 3 years old. European American mothers reported more positive emotional expression than Mainland Chinese mothers, but no difference was found in their expression of negative emotions. In their study, Brown et al. (2015) examined variability in 495 families of African American, European American, and Lumbee American Indian ethnicities, mothers and fathers' reactions and expressiveness toward children who were 4 to 10 years old. They emphasized that mothers and fathers reported similar levels of supportive reactions toward their children in African American culture, but that mothers reported more supportive reactions than fathers in the European American and Lumbee cultures. They also reported that mothers were more supportive toward girls' negative emotions than fathers across all ethnicities, while toward boys, parents' gender differences in supportive reactions to negative emotions varied depending on ethnicity. Toward European American boys, mothers reported more supportive reactions than fathers, while toward African American boys, mothers were less supportive than fathers. These studies highlighted the contextualized nature of parents' emotion socialization depending on culture and ethnicity, which should be considered in research.

It could be interesting to compare parents' emotion socialization in relation to socio-emotional abilities in TD children and atypically developing children, in two French-speaking countries, Quebec and Belgium, in considering both educational and cultural factors. Some differences between Quebec and Belgium could result in a differential impact on maternal socialization, depending on proximal and more distal factors, such as the type of support given to mothers, the positive vs negative consideration of partnerships between parents and teachers or professionals, the variability of quality of efficient practices in parents from different cultural and educational levels, the accessibility and the understanding of information about socio-emotional development in children (for example, in parents' associations, as there are fewer parents with a low educational level, they have less access to relevant information (Abdeljalil \& Changkakoti, 2009)).

Some differences in socio-emotional skills between Quebec and Belgian children, even those with similar developmental ages, could be caused by differences in the quality of intervention and education given to them in this domain, in school and services. The support given to children could differ depending on the teachers' knowledge and education on evidence-based practices targeting emotional and social competences in atypically developing children and on how to support their social learning and inclusion; there are differences in initial and continuing training, notably in evidence-based programs, in Quebec and Belgium (Abdeljalil \& Changkakoti, 2009; Potvin et al., 2018). In Quebec, a community approach, networking, and openness to socio-cultural diversity and disabilities have been implemented for several decades, in prevention and early intervention, helping families to benefit from complementary services, a good 
partnership with schools, special services, and extracurricular services (Potvin et al., 2018). For children with special needs, inclusive practices and systems in schools and in other environments vary according to cultural contexts in Quebec and Belgium (Nédélec-Trohel, Bocage \& Kalubi, 2015). In addition, relationships and partnerships between parents and schools are implemented differently in Quebec and in Belgium. Abdeljalil \& Changkakoti (2009) highlighted intercultural variations in terms of recommendations for the various players in the school field, programs to increase parental involvement in school, systems put in place to support families, the perception of respective expertise in professionals and parents (in particular, in parents with a low educational level), and the common vs uncommon educative values, norms, and strategies between families and schools. Different factors, specified by the OECD (2018), influence quality in early childhood education and care, in both center-based and family daycare settings, that contribute in a favorable or unfavorable way to children's development. A higher process quality could be associated with higher levels of children's development, notably as regards social skills. Some similarities vs differences between Quebec and Belgium in these quality factors could be more favorable or unfavorable to both children's and parents' abilities.

\section{Objectives and hypotheses of the study}

This study was based mainly on the model of Eisenberg et al. (1998a, 1998b), with the aim of formulating hypotheses about maternal reactions toward their typically developing (TD) children or children with intellectual disabilities (ID) and the selection of assessment measures. As regards the approach used for socio-emotional skills in children, the study referred to the heuristic model of social competences developed by Yeates et al. (2007), adapted by Nader-Grosbois (2011), which includes: 1) Social-cognitive processing including ToM abilities; 2) Emotion regulation in social interactions; 3 ) Social adjustment, in typical and atypical development, to guide hypotheses and select targeted measures for children with and without ID. This study was also based on the Vygotskian socio-constructivist approach of ToM development, considering the impact of interpersonal factors.

Given cultural factors that could vary in the two French-speaking countries, Quebec and Belgium, this exploratory comparative study aims first to investigate how ToM, emotion regulation, and social adjustment in Belgian and Quebec children with and without ID vary according to individual factors (age, global developmental age, the TD vs ID factor) and environmental factors, cultural and maternal factors, educational level, and emotion-related reactions. Secondly, the study aims to explore similarities vs differences between Belgian and Quebec mothers in their reactions to emotions felt by their TD children or children with ID of a similar developmental age, and the predictors. The hypotheses are as follows:

1) We expected that children's ToM, emotion regulation and social adjustment would vary depending on their global developmental age, the TD vs ID 
factor, the culture (Belgian vs Quebec) and their mothers' level of education and reactions about emotions.

2) We expected that mothers' supportive vs unsupportive reactions and specific reactions to positive and negative emotions would vary according to the children's characteristics (GDA, TD vs ID factor) and socio-emotional abilities, and depending on the culture (Belgian vs Quebec) and their own educational level.

\section{Methods}

\subsection{Participants}

Seventy-two children (41 boys and 30 girls) including 36 TD children and 36 children with ID, and their mothers, living in French-speaking areas of Belgium and in Quebec, participated in this study. The children in Quebec and Belgium all benefited from specialized education and care. Information about recruitment process and school conditions are described in the procedure section. Among the 92 parents who responded to the call, 21 participants were excluded after consideration of the exclusion and inclusion criteria.

As regards exclusion criteria, children with Williams syndrome or autistic spectrum disorder were not to be included. In fact, major deficits in Theory of Mind, a high emotional dysregulation, and weak social adjustment are present in children with autistic spectrum disorder (Nader-Grosbois \& Mazzone, 2014), and children with dual diagnosis of ID and autistic spectrum disorder. About children with Williams syndrome, they were excluded because of their specific socio-emotional profiles (Bellugi et al., 2000) and language competences (Mervis \& Velleman, 2011).

In order to benefit from specialized services, all children with ID had to be diagnosed by specialists, in accordance with the criteria of the AAIDD (American Association on Intellectual and Developmental Disabilities (Schalock et al., 2010) and DSM-V (The Diagnostic and Statistical Manual of Mental Disorders (American Association of Psychology, 2013), including a deficient intelligence quotient (IQ) and adaptive functioning. To participate, children with ID had to display an IQ between 50 and 70 and all children had to display a preschool developmental age (between 3 and 6).

The TD children were $3 \frac{1}{2}$ to 6 years old. To determine cognitive abilities and GDA in children with and without ID, Belgian researchers administered WPPSIIII to children at school, while Quebec researchers asked parents to complete the Child Development Inventory-French version (IDE) (see instruments section). These two measures made it possible to determine global developmental age (GDA) using two different approaches, namely neuropsychological and behavioral. The mean chronological age (CA) and GDA in months and the gender distribution are presented, in results section, in Table 1, for TD children in each cultural sample and in Table 2 for children with ID that were approximately 7 to 12 years old. 
To estimate maternal socio-cultural level, the mothers indicated their levels of education: 1 = primary school uncompleted; 2 = primary school; 3 = primary in special education school; $4=$ secondary school; $5=$ secondary in special education school; $6=$ apprenticeship; $7=$ bachelor's in higher education program; $8=$ master's in higher education program; $9=\mathrm{PhD}$. For regression analyses, mothers were divided into three levels of education: 1) "Low" level for mothers who had only completed primary school; 2) "Medium" for mothers who had completed special primary school, secondary school, special secondary school or an apprenticeship; 3) "High" mothers who had completed a higher education program at secondary school or university. In results section, Table 3 and Table 4 indicate the educational levels of mothers as socio-cultural information about these samples.

\subsection{Measures}

Wechsler preschool and primary scales (WPPSI-III (Wechsler, 2004)). Four subtests- "information", "vocabulary", "block design" and "matrix reasoning" of the well-known WPPSI-III were administered to Belgian children. It gives verbal and non-verbal cognitive functioning, intelligence quotient, and global developmental age.

Child Development Inventory-French version (IDE (Duyme, Capron \& Zorman, 2010; Ireton, 1992; Ireton \& Glascoe, 1995). The short form of this questionnaire is composed of 70 items, divided in eight domains (expressive and receptive language, letters and numbers abilities, social domain, autonomy, global and fine motor skills). For each item, the answers are "Yes" or "No". It allows calculating a developmental age. The interrater reliability index (parents vs teacher) is .78 and the test-retest reliability coefficient is .97 (Duyme \& Capron, 2010). A high significant correlation $(r=.83 ; p=.000)$ was obtained between developmental quotient in IDE and IQ in WPPSI-III, in the validation study (Duyme \& Capron, 2010). In the present study, IDE was completed by mothers of children in Quebec.

Theory of Mind Inventory-French version (ToMI-vf (Houssa, Mazzone, \& Nader-Grosbois, 2014; Hutchins, Prelock, \& Bonazinga, 2012). This questionnaire assesses adults' perceptions of children's ToM abilities in daily life. Through 39 items, the mothers' rate their children's comprehension of nine mental states (affective states such as emotions and desires, as well as cognitive states, including beliefs, false beliefs, intentions, attention, perception, thinking, pretend play). For each item, the mother indicates the answer on a continuum comprising "definitely not”, "probably not”, "undecided”, "probably", and "definitely". The total score ranges from 0 to 20 . Three subscores can be calculated, relating respectively to beliefs, socio-emotional states, and thoughts. Validation of the French version matched that of the original version. Moreover, the French version shows significant internal consistency $(\alpha=.94)$ and test-retest reliability $(\mathrm{r}=.86)$. 
Social adjustment scales for children-French version (EASE (Comte-Gervais et al., 2008; Hughes et al., 1997). This questionnaire measures adults' perception of children's social adjustment. It comprises 50 items, 25 items related to ToM in everyday social interactions and 25 to social skills referring to observance of social rules. For each item, the mothers estimate, on a 2-point Likert scale, the frequency of behaviors: "rarely", "relatively frequently", or "usually". The total score ranges from 0 to 98, and the two subscores, EASE-ToM and EASE-Social Skills, range from 0 to 52 and 0 to 46 respectively). The two subscales have good internal consistency, with Cronbach's alphas of .77 and .79 respectively (ComteGervais et al., 2008).

Emotion Regulation Checklist-French version (ERC-vf (Nader-Grosbois \& Mazzone, 2015; ERC, Shields \& Cicchetti, 1997)). This questionnaire assesses adults' perception of children's emotional regulation. The 24 items measure intra- and inter-personal emotional regulation and dysregulation. They correspond to the description of emotional responses and expressions of joy, anger, or empathy toward peers or adults, or of behaviors displayed in situations inducing frustration and change of activity. This measure is applicable to children aged from 3 to 12 years old with or without a development disorder. The mothers evaluate the frequency of the behavior on a 4-point Likert scale ranging from "never" to "almost always". Two raw scores can be obtained: an emotional regulation score and an emotional dysregulation score. The sum of the two raw scores gives a composite emotional regulation score. The different raw scores can be averaged. The factor analysis for the French version confirmed the two factors, "emotional regulation" and "emotional dysregulation". The internal consistency for this checklist has a Cronbach's alpha of .72 and a test-retest reliability of .92 (Nader-Grosbois \& Mazzone, 2015).

Apart from cognitive measures, none of the measures gave standards, but they provided information about strengths and weaknesses in children's profiles.

Parental Reactions to Positive and Negative Emotions (Daffe \& Nader-Grosbois, 2009). This questionnaire is an integrated version of two questionnaires, namely "Questionnaire sur les Réactions Parentales aux Emotions Positives Exprimées Par L'Enfant" (QRPEPE (Ladouceur, Reid, \& Jacques, 2002)) and "Coping with Children's Negative Emotions Scale"-French version (CCNES-vf (Coutu et al., 2002; Fabes et al., 2002). The questionnaire presents eight hypothetical scenarios in which a child feels negative (fear, sadness, and anger) or positive emotions (joy). For the scenarios related to negative emotions, six parental reactions are suggested (encouragement of emotional expression, comforting, problem-focused responses, distress, minimizing, and punitive response). For the positive scripts, four parental reactions are proposed (reprimand, discomfort, socialization, and encouragement). Examples of item are presented in appendixes A and B. The mothers indicate, for each item, to what extent they use different strategies on a 7-point Likert scale ranging from "very unlikely" to "very likely". The factor analysis of this questionnaire, validated on 328 parents of TD children, distin- 
guished two subscales, supportive and unsupportive reactions for positive and negative emotions. Scores for supportive and unsupportive reactions and a score for each type of reaction can be calculated. Cronbach's alpha is .78 and .81 for negative scenarios and .77 and .62 for the joy scripts.

\subsection{Procedure}

Before starting the recruitment, the research procedure was approved, by the Ethics Commission of the Psychological Sciences Research Institute of UCLouvain in Belgium and, in Canada, by the Research Ethics Board of the Université du Québec à Montréal, and the Joint Research Ethics Committee (CERC DITSA) for Quebec ID rehabilitation centers.

In Belgium, TD children were recruited in primary schools, and children with ID in special primary schools, by means of a letter summarizing the aims of the research and modalities of participation and a consent document for parents. In Quebec, mothers of TD children were recruited via social media, and information was posted in various parents' groups as well as in a poster describing the study and including a link to the online consent document and all questionnaires; mothers of children with ID were also contacted by means of a letter addressed to various ID rehabilitation centers, community organizations, and two special schools in Montréal.

Children with ID from Quebec and their families benefit from the support provided by the "Centre de réadaptation en déficience intellectuelle et en troubles envahissants du développement" (CRDITED) whose priority is their social inclusion. The sharing of scientific knowledge and education about best evidencebased practices by university experts is well organized, and partnerships between researchers and professional teams contribute to improving the quality of their services.

In Belgium, on the other hand, ID children participate in special school activities offered by specialized "type 2" teaching adapted for moderate intellectual disability. There are no specific partnerships with universities' continuing education departments; however, teachers have access to continuing education. Most parents have very limited or no dialog with teachers because their ID children are in internships or semi-boarding schools.

All the measures were questionnaires filled out by mothers, in paper or online version, according to their choice, and for the Belgian sample, they could be completed in the presence of a searcher, if preferred. $20 \%$ of mothers, mostly those with a low education level, requested this option.

By responding to the questionnaires, the mothers gave their perception about their child's ToM, social adjustment, and emotional regulation, and reported their own emotion-related socialization behaviors (ERSBs), namely their reactions to the child's emotions. Only the WPPSI-III was administered to Belgian children, in a quiet room at school. The study was conducted during 4 years (recruitment to data analysis). 
Finally, complete content and organizational editing before formatting. Please take note of the following items when proofreading spelling and grammar.

\section{Results}

\subsection{Descriptive Statistics and Comparison between Cultural Groups of Children's Characteristics and Socio-Emotional Competences}

Table 1 and Table 2 present the descriptive statistics and cross-cultural comparison of Belgian and Quebec samples of TD and ID children respectively, concerning their individual characteristics, their gender distribution, chronological age (CA), global developmental age (GDA), and also their abilities in ToM, social adjustment, and emotion regulation.

Considering individual characteristics, the TD children in the two cultural groups do not differ in terms of gender, CA, and GDA, so they are well matched on their two variables. As regards socio-emotional competences, the TD children of the two cultural groups show no significant difference in their ToM abilities and in their low level of emotional dysregulation, but they differ significantly in their social skills and level of emotional regulation, which are better in the Quebec sample. The two cultural ID groups do not differ significantly in ToM, social adjustment, or emotional regulation or dysregulation.

Table 1. Means, standard deviation, and comparison of TD children's characteristics and socio-emotional competences in Belgian and Quebec samples.

\begin{tabular}{|c|c|c|c|c|}
\hline & $\begin{array}{c}\text { Belgian sample } \\
\begin{array}{c}M(\mathrm{SD}) \\
n=18\end{array}\end{array}$ & $\begin{array}{c}\text { Quebec sample } \\
M(\mathrm{SD}) \\
n=18\end{array}$ & $X^{2} / t$ & $d$ \\
\hline \multicolumn{5}{|l|}{ TD Children's characteristics } \\
\hline Sex (\% boys) & $55 \%$ & $61 \%$ & .73 & \\
\hline Chronological age (months) & $57.97(22.99)$ & $48.33(8.51)$ & 1.67 & \\
\hline Global developmental age (months) & $53.38(12.16)$ & $50.92(7.19)$ & .74 & \\
\hline \multicolumn{5}{|c|}{ TD Children's socio-emotional competences } \\
\hline ToMI-Total $(\max =20)$ & $14.28(2.68)$ & $14.45(2.15)$ & -.20 & \\
\hline ToMI-“Thoughts" Factor 1 & $12.01(3.21)$ & $11.55(3.16)$ & .44 & \\
\hline ToMI-“Socio-emotional” Factor 2 & $15.87(2.22)$ & $17.28(1.99)$ & -1.99 & .67 \\
\hline ToMI-“Beliefs" Factor 3 & $16.76(3.04)$ & $17.10(1.91)$ & -.40 & \\
\hline EASE total $(\max =98)$ & $63.46(18.82)$ & $77.83(10.87)$ & $-2.69^{*}$ & .93 \\
\hline EASE-ToM $(\max =52)$ & $28.61(11.06)$ & $35.78(6.99)$ & -2.06 & .77 \\
\hline EASE-Social Skills $(\max =46)$ & $34.85(8.06)$ & $42.05(4.09)$ & $-3.27^{\star \star}$ & 1.13 \\
\hline Emotion Regulation & $2.53(.17)$ & $3.39(.35)$ & $-6.66^{* * *}$ & 3.12 \\
\hline Emotion Dysregulation & $1.96(.11)$ & $1.77(.36)$ & .91 & \\
\hline
\end{tabular}

${ }^{\mathrm{a}}$ Notes: $M=$ mean, $\mathrm{SD}=$ standard deviation, $\mathrm{TD}=$ typically developing, ${ }^{*} p<.05,{ }^{* *} p<.01 ;{ }^{* *} p<.001$. 
Table 2. Means, standard deviation, and comparison of ID children's characteristics and socio-emotional competences in Belgian and Quebec samples.

\begin{tabular}{|c|c|c|c|c|}
\hline & $\begin{array}{c}\text { Belgian sample } \\
\begin{array}{c}M(\mathrm{SD}) \\
n=18\end{array}\end{array}$ & $\begin{array}{c}\text { Quebec sample } \\
M(\mathrm{SD}) \\
n=18\end{array}$ & $X^{2} / t$ & $d$ \\
\hline \multicolumn{5}{|l|}{ ID Children's characteristics } \\
\hline Sex (\% boys) & $72 \%$ & $41 \%$ & .06 & \\
\hline Chronological age (in months) & $99.94(24.29)$ & $125(17.55)$ & $-3.51^{* * *}$ & 1.18 \\
\hline Global developmental age (in months) & $53.14(11.73)$ & $49.71(7.79)$ & 1.01 & \\
\hline \multicolumn{5}{|c|}{ ID Children's socio-emotional competences } \\
\hline ToMI-Total $(\max =20)$ & $12.36(2.59)$ & $13.04(3.38)$ & -.57 & \\
\hline ToMI-“Thoughts” Factor 1 & $10.68(3.44)$ & $10.68(4.55)$ & -.01 & \\
\hline ToMI-“Socio-emotional” Factor 2 & $14.61(2.25)$ & $15.20(2.76)$ & -.63 & \\
\hline ToMI-“Beliefs" Factor 3 & $14.13(4.09)$ & $15.39(3.70)$ & -.85 & \\
\hline EASE total $(\max =98)$ & $56.53(19.70)$ & $46.75(21.64)$ & 1.35 & \\
\hline EASE-ToM $(\max =52)$ & $27.41(10.37)$ & $20.44(10.79)$ & 1.89 & \\
\hline EASE-Social Skills $(\max =46)$ & $29(9.92)$ & $26.31(11.12)$ & .73 & \\
\hline Emotion Regulation & $2.89(.41)$ & $3.01(.57)$ & -.64 & \\
\hline Emotion Dysregulation & $2.08(.36)$ & $2.07(.36)$ & .09 & \\
\hline
\end{tabular}

${ }^{a}$ Notes: $M=$ mean, $\mathrm{SD}=$ standard deviation, $\mathrm{ID}=$ intellectual disabilities, ${ }^{* * *} p<.001$.

The children with ID in both cultural groups present a similar GDA; however, the comparison of their CA indicates that the Quebec children are older than the Belgian children, so they are well matched on their GDA.

Moreover, an ANOVA was performed for GDA for the four groups, which confirmed the good matching on GDA $(F=.55 ; p=.65)$.

A two-way MANOVA, applied with the two EASE subscores("EASE-Social Skills" and "EASE-ToM") as dependent variables, indicated that the status of ID vs TD in children is related to social adjustment $\left(F=15.87 ; p=.000 ; \eta^{2}=.35\right)$, while the cultural factor is not linked with social adjustment $(F=2.87 ; p=.065$; $\eta^{2}=.09$ ). Therefore, ID children presented lower social abilities, in line with their diagnosis.

\subsection{Descriptive Statistics and Comparison between Cultural Groups of Mothers' Educational Levels, Emotion-Related Reactions}

In the entire sample, $17 \%$ of mothers had only completed primary school; $39 \%$ of mothers had completed secondary school, special secondary school, or an apprenticeship; $38 \%$ of mothers had completed a higher education program. The distribution of educational levels of Belgian and Quebec mothers of TD and ID children, and descriptive statistics were specified in Table 3. 
Table 3. Percentages and descriptive statistics of educational level in Belgian and Quebec mothers' of TD or ID children.

\begin{tabular}{|c|c|c|c|c|c|c|c|c|}
\hline & \multicolumn{4}{|c|}{ Mothers of TD children } & \multicolumn{4}{|c|}{ Mothers of ID children } \\
\hline & Belgian & Quebec & & & Belgian & Quebec & & \\
\hline \multicolumn{9}{|l|}{ Percentages in distinct educational level } \\
\hline Primary school & $11 \%$ & $0 \%$ & & & $55 \%$ & $0 \%$ & & \\
\hline Secondary school or an apprenticeship & $44 \%$ & $17 \%$ & & & $28 \%$ & $71 \%$ & & \\
\hline High education & $28 \%$ & $83 \%$ & & & $11 \%$ & $29 \%$ & & \\
\hline Descriptive statistics of educational level & $M(\mathrm{SD})$ & $M(\mathrm{SD})$ & $t$ & $d$ & $M(\mathrm{SD})$ & $M(\mathrm{SD})$ & $t$ & $d$ \\
\hline Mothers' level of education $(\max =9)$ & $5.33(1.5)$ & $7.22(1.11)$ & $-4.15^{\star *}$ & 1.43 & $3.29(2.05)$ & $5.24(1.79)$ & $-2.94^{\star}$ & 1.01 \\
\hline
\end{tabular}

${ }^{\mathrm{a}}$ Notes: $M=$ mean, $\mathrm{SD}=$ standard deviation, $\mathrm{TD}=$ typically developing; $\mathrm{ID}=$ intellectual disabilities, ${ }^{*} p<.01 ;{ }^{* *} p=.001$.

The mothers of TD children and of ID children differ significantly in their educational levels, in the sense that the Quebec mothers have a higher level than the Belgian mothers.

Table 4 and Table 5 present the descriptive statistics of mothers' supportive and unsupportive reactions toward their child's emotions, in the Belgian and Quebec samples, respectively toward TD and ID children.

Table 4. Descriptive statistics related to mothers' emotion-related reactions toward their TD children in Belgian and Quebec samples.

\begin{tabular}{|c|c|c|}
\hline Mothers' reactions to their TD child's emotions & $\begin{array}{c}\text { Belgian sample } \\
M(\mathrm{SD}) \\
n=18\end{array}$ & $\begin{array}{c}\text { Quebec sample } \\
M(\mathrm{SD}) \\
n=18\end{array}$ \\
\hline Supportive reactions to negative emotions & $4.94(.66)$ & $5.51(.59)$ \\
\hline Comforting & $4.89(.77)$ & $5.19(.55)$ \\
\hline Problem-focused & $5.62(.89)$ & $6.05(.73)$ \\
\hline Encouragement of expression of emotion & $4.31(1.09)$ & $5.31(1.02)$ \\
\hline Unsupportive reactions to negative emotions & $2.52(.56)$ & $2.67(.52)$ \\
\hline Distress & $2.44(.69)$ & $2.29(.62)$ \\
\hline Punition & $1.94(.75)$ & $2.19(.48)$ \\
\hline Minimizing responses & $3.19(.72)$ & $3.53(.87)$ \\
\hline Supportive reactions to positive emotions & $4.69(.70)$ & $5.32(.68)$ \\
\hline Socialization & $4.92(1.83)$ & $6.19(.88)$ \\
\hline Encouragement & $4.46(1.45)$ & $4.44(.95)$ \\
\hline Unsupportive reactions to positive emotions & $2.92(1.38)$ & $3.24(1.17)$ \\
\hline Reprimand & $3.67(1.69)$ & $3.56(1.40)$ \\
\hline Discomfort & $2.17(1.34)$ & $2.92(1.19)$ \\
\hline
\end{tabular}

${ }^{\mathrm{a}}$ Notes: $M=$ mean, $\mathrm{SD}=$ standard deviation, $\mathrm{TD}=$ typically developing. 
Table 5. Descriptive statistics related to mothers' emotion-related reactions toward their children with ID, in Belgian and Quebec samples.

\begin{tabular}{|c|c|c|}
\hline Mothers' reactions to their ID child's emotions & $\begin{array}{c}\text { Belgian sample } \\
M(\mathrm{SD}) \\
n=18\end{array}$ & $\begin{array}{c}\text { Quebec sample } \\
\begin{array}{c}M(\mathrm{SD}) \\
n=18\end{array}\end{array}$ \\
\hline Supportive reactions to negative emotions & $5.28(.59)$ & $5.53(.54)$ \\
\hline Comforting & $5.76(.84)$ & $5.07(.91)$ \\
\hline Problem-focused & $5.51(.89)$ & $6.03(.68)$ \\
\hline Encouragement of expression of emotion & $4.57(.55)$ & $5.48(.97)$ \\
\hline Unsupportive reactions to negative emotions & $4.06(1.08)$ & $2.63(.54)$ \\
\hline Distress & $3.06(1.49)$ & $2.31(.69)$ \\
\hline Punition & $3.95(1.35)$ & $2.12(.64)$ \\
\hline Minimizing responses & $5.23(.96)$ & $3.45(1.10)$ \\
\hline Supportive reactions to positive emotions & $5.01(1.04)$ & $5.15(.89)$ \\
\hline Socialization & $5.39(1.68)$ & $5.53(1.77)$ \\
\hline Encouragement & $4.64(1.26)$ & $4.76(1.35)$ \\
\hline Unsupportive reactions to positive emotions & $3.69(1.67)$ & $3.43(1.43)$ \\
\hline Reprimand & $4.22(2.03)$ & $4(1.42)$ \\
\hline Discomfort & $3.19(1.52)$ & $2.85(1.81)$ \\
\hline
\end{tabular}

${ }^{a}$ Notes: $M=$ mean, $\mathrm{SD}=$ standard deviation, $\mathrm{ID}=$ intellectual disabilities.

To explore the link between cultural factor with mothers' reactions of socialization, considering as covariate maternal level of education, one-way MANCOVAs were applied. Table 6 and Table 7 presented multivariate effects respectively for TD samples.

Concerning TD groups (in Table 6), one-way MANCOVAs on supportive and unsupportive reactions scores toward positive and negative emotions showed no effect of cultural and educational level factors on each global score of maternal reaction.

Table 6. One-way MANCOVA between culture and maternal supportive and unsupportive reactions to positive and negative emotions, with as covariate maternal educational level, in TD samples

\begin{tabular}{ccccc}
\hline Effects & Pillai's Trace & $F$ & $\boldsymbol{p}$ & $\boldsymbol{\eta}^{2}$ \\
\hline Maternal Level of Education & .096 & .721 & .585 & .096 \\
Culture & .134 & 1.046 & .402 & .134 \\
\hline
\end{tabular}

Similarly, one-way MANCOVAs on each specific supportive and unsupportive reaction toward positive and negative emotions (see Table 7) indicated no effect of cultural and educational level factors on specific reaction, except for socialization of positive emotions. In fact, an effect of cultural factor was obtained on socialization $\left(F=5.97 ; p<.05, \eta^{2}=.16\right)$. 
Table 7. One-way MANCOVA between culture and 10 specific maternal reactions to positive and negative emotions, with as covariate maternal educational level, in TD samples.

\begin{tabular}{ccccc}
\hline Effects & Pillai's Trace & $\boldsymbol{F}$ & $\boldsymbol{P}$ & $\boldsymbol{\eta}^{2}$ \\
\hline Maternal Level of Education & .160 & .399 & .932 & .160 \\
Culture & .386 & 1.323 & .282 & .386 \\
\hline
\end{tabular}

As regards ID groups, were applied first MANCOVAs on supportive and unsupportive reactions scores toward positive and negative emotions (see Table 8). Results showed an effect of culture and tests of between-subjects demonstrated effects of culture on unsupportive reactions toward negative emotions $(F=$ 22.63; $\left.p=.000, \eta^{2}=.42\right)$.

Table 8. One-way MANCOVA between culture and maternal supportive and unsupportive reactions to positive and negative emotions, with as covariate maternal educational level, in ID samples.

\begin{tabular}{ccccc}
\hline Effects & Pillai's Trace & $\boldsymbol{F}$ & $\boldsymbol{p}$ & $\boldsymbol{\eta}^{2}$ \\
\hline Maternal Level of Education & .068 & .511 & .728 & .068 \\
Culture & .515 & 7.442 & .000 & .515 \\
\hline
\end{tabular}

Secondly, MANCOVAs were applied on each specific reaction to positive and negative emotions (see Table 9). Results showed an effect of culture. Tests of between-subjects demonstrated effects of culture on punition $(F=23.81 ; p$ $\left.=.000, \eta^{2}=.43\right)$, minimization $\left(F=25.26 ; p=.000, \eta^{2}=.45\right)$, comforting $(F=$ 4.25; $\left.p<.05, \eta^{2}=.12\right)$ and encouragement $\left(F=8.68 ; p<.01, \eta^{2}=.22\right)$ toward negative emotions.

Table 9. One-way MANCOVA between culture and level and 10 specific maternal reactions to positive and negative emotions, with as covariate maternal educational level, in ID samples.

\begin{tabular}{ccccc}
\hline Effects & Pillai's Trace & $F$ & $p$ & $\eta^{2}$ \\
\hline Maternal Level of Education & .205 & .566 & .823 & .205 \\
Culture & .755 & 6.796 & .000 & .755 \\
\hline
\end{tabular}

\subsection{Variability of Theory of Mind, Social Adjustment, and Emotion Regulation in TD and ID Children According to Individual Children's and Mothers' Factors and Maternal Reactions}

Preliminary correlational analyses using Spearman coefficients were performedfor each group, between CA, GDA, maternal educational levels, and scores in ToMI-Total, EASE, and ERC in emotion regulation and dysregulation. In the Belgian sample, the TD children's GDA is positively linked to their ToMI-Beliefs $(r=.507, p>.05)$. In Belgian children with ID, the ToMI-Beliefs is positively correlated with CA $(r=602, p<.05)$, the social adjustment in EASE Total is po- 
sitively correlated with CA $(\mathrm{r}=.518, p<.05)$, and GDA $(r=.504, p<.05)$, and EASE-Social Skills are positively linked to CA $(r=.534, p<.05)$ and GDA $(r$ $=.543, p<.05)$. In Quebec TD children, no significant correlation is obtained between all socio-emotional abilities and their CA and GDA respectively. In Quebec children with ID, no significant correlation is obtained between their CA and all socio-emotional competences in ToM, emotion regulation, and social adjustment scores; however, positive links are obtained between their GDA and ToMI-Total $(r=.670, p<.01)$, specifically ToMI-Thoughts $(r=.609, p<.01)$, ToMI-Socioemotional $(r=.519, p<.05)$, ToMI-Beliefs $(r=.622, p<.01)$. In the Belgian sample only, the mothers' educational level is positively and significantly correlated with abilities of TD children in EASE total $(r=.642, p<.05)$, and specifically in EASE-ToM $(r=.644, p<.05)$, while in the Quebec sample, the mothers' educational level is correlated positively and significantly with ToM socio-emotional abilities in TD children $(r=.495, p<.05)$.

Secondly, in order to explore the extent to which individual characteristics of children (GDA and the TD vs ID factor), of mothers (educational level), the culture (Belgian vs Quebec), and mothers' ERSBs (reactions) contribute to predicting a percentage of the variance in the children's socio-emotional competences, linear regression analyses with a stepwise method were performed. In Step 1, children's characteristics (GDA and TD vs ID group), the culture, and the level of education of mothers (low, medium, high) were entered. Then, in Step 2, the different supportive and unsupportive reactions to negative and positive emotions were entered, namely comforting, problem-focused responses, encouragement of emotional expression, socialization, encouragement, distress, punitive reactions, minimizing, reprimand, and discomfort. The variance inflation factor (VIF) was used to assess multicollinearity. For each model, no multicollinearity was observed. Table 10 and Table 11 present the results concerning significant predictors of children's socio-emotional competences as reported by mothers.

Table 10. Predictors of children's theory of mind competences depending on individual characteristics and mothers' educational level and emotion-related reactions.

\begin{tabular}{|c|c|c|c|c|c|}
\hline \multicolumn{6}{|c|}{ Children' Theory of Mind competences } \\
\hline \multirow{2}{*}{ Predictors } & \multicolumn{5}{|c|}{ ToMI-Total } \\
\hline & $B$ & $S E / B$ & $\beta$ & $R^{2}$ adj & $F$ \\
\hline Model M1a & & & & .196 & $15.912^{* * * *}$ \\
\hline \multirow[t]{2}{*}{ GDA } & .131 & .033 & $.458^{* * * *}$ & & \\
\hline & \multicolumn{5}{|c|}{ ToMI-“Thoughts” Factor 1} \\
\hline Predictors & $B$ & $S E / B$ & $\beta$ & $R^{2}$ adj & $F$ \\
\hline Model M1a & & & & .219 & $18.063^{* * * *}$ \\
\hline GDA & .181 & .043 & $.481^{\star * * *}$ & & \\
\hline
\end{tabular}




\section{Continued}

\begin{tabular}{|c|c|c|c|c|c|}
\hline \multirow{2}{*}{ Predictors } & \multicolumn{5}{|c|}{ ToMI-“Socioemotional” Factor 2} \\
\hline & $B$ & $S E / B$ & $\beta$ & $R^{2} a d j$ & $F$ \\
\hline Model M1b & & & & .116 & $8.966^{* *}$ \\
\hline Education Level_High & 1.810 & .605 & $.361^{* *}$ & & \\
\hline Model M2b & & & & .167 & $7.132^{\star * *}$ \\
\hline Education Level_High & 1.672 & .590 & $.333^{* *}$ & & \\
\hline GDA & .066 & .030 & $.256^{*}$ & & \\
\hline \multirow{2}{*}{ Predictors } & \multicolumn{5}{|c|}{ ToMI-“Beliefs” Factor 3} \\
\hline & $B$ & $S E / B$ & $\beta$ & $R^{2}$ adj & $F$ \\
\hline Model M1c & & & & .198 & $16.094^{* * * *}$ \\
\hline GDA & .161 & .040 & $.460^{\star * * *}$ & & \\
\hline Model M2c & & & & .262 & $11.827^{\star * * *}$ \\
\hline GDA & .153 & .039 & $.439^{* * * *}$ & & \\
\hline ID or TD children & -1.843 & .742 & $-.274^{*}$ & & \\
\hline Model M3c & & & & .299 & $9.685^{\star \star \star *}$ \\
\hline GDA & .171 & .039 & $.489^{\star * * *}$ & & \\
\hline ID or TD children & -1.879 & .723 & $.279^{*}$ & & \\
\hline Culture & 1.516 & .745 & $.224^{*}$ & & \\
\hline
\end{tabular}

${ }^{a}$ Notes. $-\mathrm{E}=$ negative emotions, $+\mathrm{E}=$ positive emotions, $\mathrm{GDA}=$ Global developmental age, $\mathrm{TD}=$ typically developing, ID $=$ intellectual disabilities; ${ }^{*} p<.05,{ }^{* *} p<.01,{ }^{* *} p=.001,{ }^{* * *} p=.000$.

Concerning predictors of ToM abilities (Table 10), children's GDA explained $21 \%$ of the variance in these children's competences (total ToMI score).

Specifically, children's GDA explained $23 \%$ of the variance in ToMI-Thoughts. $19 \%$ of the variance in ToMI-Socioemotional was explained by Model M2b including mothers' educational level corresponding to higher education $(\beta=.360$; $p \leq .01)$ and children's GDA. $29 \%$ of the variance in ToMI-Beliefs was explained by Model M3c, which included children's GDA, TD vs ID group and culture.

Table 11. Predictors of children's social adjustment and emotion regulation depending on individual characteristics and mothers' educational level and emotion-related reactions.

\begin{tabular}{|c|c|c|c|c|c|}
\hline \multicolumn{6}{|c|}{ Children's socio-emotional competences } \\
\hline \multirow{2}{*}{ Predictors } & \multicolumn{5}{|c|}{ EASE Total } \\
\hline & $B$ & $S E / B$ & $\beta$ & $R^{2}$ adj & $F$ \\
\hline Model M1d & & & & .248 & $20.778^{* * * *}$ \\
\hline ID or TD children & -21.248 & 4.661 & $-.510^{\star * * *}$ & & \\
\hline Model M2d & & & & .306 & $14.202^{* * * *}$ \\
\hline ID or TD children & -22.945 & 4.533 & $-.551^{\star * * *}$ & & \\
\hline Reprimand to $+\mathrm{E}$ & 3.364 & 1.385 & $.264^{*}$ & & \\
\hline
\end{tabular}




\section{Continued}

\begin{tabular}{|c|c|c|c|c|c|}
\hline \multirow{2}{*}{ Predictors } & \multicolumn{5}{|c|}{ EASE-ToM } \\
\hline & $B$ & $S E / B$ & $\beta$ & $R^{2}$ adj & $F$ \\
\hline Model M1e & & & & .169 & $13.181^{\star * *}$ \\
\hline ID or TD children & -9.291 & 2.559 & $-.427^{\star \star \star}$ & & \\
\hline Model M2e & & & & .221 & $9.501^{\star * * *}$ \\
\hline ID or TD children & -10.150 & 3.288 & $-.467^{\star * * *}$ & & \\
\hline Reprimand to $+\mathrm{E}$ & 1.703 & .766 & $.256^{*}$ & & \\
\hline \multirow{2}{*}{ Predictors } & \multicolumn{5}{|c|}{ EASE-Social Skills } \\
\hline & $B$ & $S E / B$ & $\beta$ & $R^{2}$ adj & $F$ \\
\hline Model M1f & & & & .321 & $29.315^{* * * *}$ \\
\hline ID or TD children & -12.017 & 2.220 & $-.576^{\star * * *}$ & & \\
\hline Model M2f & & & & .381 & $19.448^{* * * *}$ \\
\hline ID or TD children & -12.875 & 2.145 & $-.617^{* * * *}$ & & \\
\hline Reprimand to $+\mathrm{E}$ & 1.700 & .655 & $.267^{*}$ & & \\
\hline Model M3f & & & & .417 & $15.293^{* * * *}$ \\
\hline ID or TD children & -13.152 & 2.085 & $-.631^{\star * * *}$ & & \\
\hline Reprimand to $+\mathrm{E}$ & 1.833 & .639 & $.288^{\star}$ & & \\
\hline Encouragement to $-\mathrm{E}$ & 2.221 & 1.038 & $.212^{*}$ & & \\
\hline ModelM4f & & & & .448 & $13.171^{* * * *}$ \\
\hline ID or TD children & -14.672 & 2.160 & $-.703^{\star * * *}$ & & \\
\hline Reprimand to $+\mathrm{E}$ & 1.773 & .622 & $.278^{\star}$ & & \\
\hline Encouragement to $-\mathrm{E}$ & 2.724 & 1.039 & $.260^{\star}$ & & \\
\hline Punition to -E & 1.918 & .934 & $.216^{*}$ & & \\
\hline \multirow{2}{*}{ Predictors } & \multicolumn{5}{|c|}{ ERC Emotion Regulation } \\
\hline & $B$ & $S E / B$ & $\beta$ & $R^{2}$ adj & $F$ \\
\hline Model M1g & & & & .115 & $7.742^{* *}$ \\
\hline Culture & .371 & .133 & $.363^{* *}$ & & \\
\hline \multirow{2}{*}{ Predictors } & \multicolumn{5}{|c|}{ ERC Emotion Dysregulation } \\
\hline & $B$ & $S E / B$ & $\beta$ & $R^{2} a d j$ & $F$ \\
\hline Model M1h & & & & .123 & $8.311^{* *}$ \\
\hline ID or TD children & .282 & .098 & $.374^{* *}$ & & \\
\hline
\end{tabular}

${ }^{a}$ Notes. $-\mathrm{E}=$ negative emotions, $+\mathrm{E}=$ positive emotions, GDA $=$ Global developmental age, $\mathrm{TD}=$ typically developing, ID $=$ intellectual disabilities; ${ }^{*} p<.05,{ }^{* *} p<.01,{ }^{* * *} p=.001,{ }^{* * * *} p=.000$.

Regarding predictors of social adjustment (Table 11), 30\% of the variance in the EASE total score was explained by Model M2d including TD vs ID group and mothers' reprimand in response to positive emotions. Looking at predictors of EASE subscores, $22 \%$ of the variance in EASE-ToM (social adjustment in social interactions requiring understanding of mental states) was explained by the TD vs ID group and by reprimand to positive emotions. On the other hand, 44\% of the variance in EASE-Social Skills (social adjustment in social situations requiring the understanding of social conventions or rules) was explained by Model M4f, 
which included TD vs ID factor, mothers' reprimand in response to positive emotion, encouragement in response to negative emotions and punitive reaction to negative emotions.

In addition, culture explained $11 \%$ of the variance in children's emotion regulation. The TD vs ID group explained $12 \%$ of the variance in children's emotion dysregulation (see Table 11).

\subsection{Variability of Mothers' Reactions According to Individual Factors in Children and Mothers, and Socio-Emotional Competences in Children}

To investigate the extent to which individual factors in children and mothers, their culture, and the children's socio-emotional competences could predict a percentage of the variance in mothers' ERSBs (reactions), linear regression analyses with a stepwise method were performed. In Step 1, children's characteristics (GDA and the TD vs ID group), the culture (Belgian vs Quebec), and the level of education of mothers (low, medium, high) were entered. Then, in Step 2, the ToMIsubscores (namely ToMI-Thoughts, ToMI-Socioemotional, ToMI-Beliefs subscores), the EASE subscores (related to EASE-ToM or EASE-Social Skills), and the two ERC subscores (emotional regulation and dysregulation) were entered. The variance inflation factor (VIF) was used to assess multicollinearity. For each model, no multicollinearity was observed. Table 12 presents the results concerning significant predictors of mothers' ERSBs.

Regarding global scores, $31 \%$ of the variance in unsupportive reactions to negative emotions was explained by culture. Concerning reactions to positive emotions, mothers' low educational level explained $7 \%$ of the variance in unsupportive reactions, while $12 \%$ of the variance in supportive reactions was explained by Model M2b, which included ToMI-Beliefs, and ToMI-Thoughts.

Concerning specific reactions, the cultural factor explained $8 \%, 28 \%$, and $14 \%$ respectively of the variance in mothers' distress, minimization, and encouragement toward their children's negative emotions. $34 \%$ of the variance in mothers' punitive reactions to their children's negative emotions were explained by Model M2e, including culture and children's GDA. Mothers' low educational level explained $6 \%$ of the variance in their reprimand reactions to children's positive emotions.

Table 12. Predictors of mothers' reactions depending on TD and ID children's and mothers' individual characteristics and children's socio-emotional competences.

\begin{tabular}{cccccc}
\hline & \multicolumn{5}{c}{ Maternal reactions to their child's emotions } \\
\hline \multirow{5}{*}{ Predictors } & \multicolumn{5}{c}{ Unsupportive reactions to negative emotions } \\
\cline { 2 - 6 } & $B$ & $S E / B$ & $\beta$ & $R^{2}$ adj & $F$ \\
\hline $\begin{array}{c}\text { Model M1a } \\
\text { Culture }\end{array}$ & -.571 & .239 & $-1.141^{* *}$ & .312 & $22.761^{* * * *}$ \\
\hline
\end{tabular}




\section{Continued}

\begin{tabular}{|c|c|c|c|c|c|}
\hline \multirow{2}{*}{ Predictors } & \multicolumn{5}{|c|}{ Supportive reactions to positive emotions } \\
\hline & $S E / B$ & & $\beta$ & $R^{2}$ adj & $F$ \\
\hline Model M1b & & & & .062 & $4.199^{*}$ \\
\hline ToMI-“Beliefs" Factor 3 & .066 & .032 & $.286^{*}$ & & \\
\hline Model M2b & & & & .123 & $4.379^{*}$ \\
\hline ToMI-“Beliefs" Factor 3 & .110 & .038 & $.474^{\star *}$ & & \\
\hline ToMI-“Thought” Factor 1 & -.072 & .035 & $-.336^{*}$ & & \\
\hline \multirow{2}{*}{ Predictors } & \multicolumn{5}{|c|}{ Unsupportive reactions to positive emotions } \\
\hline & $B$ & $S E / B$ & $\beta$ & $R^{2}$ adj & $F$ \\
\hline Model M1c & & & & .071 & $4.683^{*}$ \\
\hline Education Level_Low & 1.123 & .519 & $.301^{*}$ & & \\
\hline \multirow{2}{*}{ Predictors } & \multicolumn{5}{|c|}{ Distress to $-\mathrm{E}$} \\
\hline & $B$ & $S E / B$ & $\beta$ & $R^{2}$ adj & $F$ \\
\hline Model M1d & & & & .086 & $5.519^{*}$ \\
\hline Culture & -.679 & .289 & $-.324^{*}$ & & \\
\hline \multirow{2}{*}{ Predictors } & \multicolumn{5}{|c|}{ Punition to $-\mathrm{E}$} \\
\hline & $B$ & $S E / B$ & $\beta$ & $R^{2}$ adj & $F$ \\
\hline Model M1e & & & & .279 & $19.602^{* * * *}$ \\
\hline Culture & -1.320 & .298 & $-.543^{* \star * *}$ & & \\
\hline Model M2e & & & & .345 & $13.641^{\star * * *}$ \\
\hline Culture & -1.457 & .290 & $-.599^{* * * *}$ & & \\
\hline GDA & -.036 & .015 & $-.285^{\star}$ & & \\
\hline \multirow{2}{*}{ Predictors } & \multicolumn{5}{|c|}{ Minimization to $-\mathrm{E}$} \\
\hline & $B$ & $S E / B$ & $\beta$ & $R^{2} a d j$ & $F$ \\
\hline Model M1f & & & & .283 & $19.944^{\star * \star *}$ \\
\hline Culture & -1.480 & .331 & $-.546^{\star * * *}$ & & \\
\hline \multirow{2}{*}{ Predictors } & \multicolumn{5}{|c|}{ Encouragement to $-\mathrm{E}$} \\
\hline & $B$ & $S E / B$ & $\beta$ & $R^{2}$ adj & $F$ \\
\hline $\begin{array}{l}\text { Model M1g } \\
\text { Culture }\end{array}$ & .839 & .282 & $.398^{* *}$ & .141 & $8.851^{\star *}$ \\
\hline \multirow{2}{*}{ Predictors } & \multicolumn{5}{|c|}{ Reprimand to $+\mathrm{E}$} \\
\hline & $B$ & $S E / B$ & $\beta$ & $R^{2} a d j$ & $F$ \\
\hline Model M1h & & & & .066 & $4.367^{*}$ \\
\hline Education Level_Low & 1.226 & .587 & $.292^{*}$ & & \\
\hline
\end{tabular}

${ }^{\mathrm{a}}$ Notes: $-E=$ negative emotions, $+E=$ positive emotions, GDA $=$ Global developmental age; ${ }^{\star} p<.05$, ${ }^{* *} p<.01,{ }^{* * *} p=.001,{ }^{* * * *} p=.000$.

\section{Discussion}

Comparison of socio-emotional abilities in Belgian and Quebec TD and ID children and individual, maternal, cultural factors

The present comparative study aims first to investigate how socio-emotional competences in French-speaking Belgian and Quebec children with and without 
ID vary according to individual factors (age, global developmental age, the TD vs ID factor), environmental factors and the culture, as well as maternal factors such as educational level and emotion-related reactions. The first hypothesis postulated that children's ToM, emotion regulation, and social adjustment vary according to their global developmental age, the TD vs ID factor, the culture(Belgian vs Quebec), and their mothers' level of education and reactions to emotions.

About the cross-cultural variability in socio-emotional competences of children (matched on their GDA), the comparative analyses showed nuanced results between TD children and children with ID. The TD children in the two cultural groups differ significantly in their social skills, and in their emotion regulation competences, which are better in Quebec children. However, both TD groups have similar abilities in ToM and displayed low emotional dysregulation. No significant difference in ToM, social adjustment, or emotional regulation or dysregulation was found between the two cultural ID groups. It seems that, in TD preschoolers only, there is a cross-cultural variability in their social adjustment in reference to cultural conventions and rules, and in their abilities to regulate emotions adequately, in the sense that Quebec preschoolers are perceived by their mothers as being more adjusted and able to regulate emotions more easily than Belgian children.

In addition, as regards children's individual factors, a difference appeared in correlations between CA, GDA, and specific socio-emotional competences in the two cultural groups with and without ID. In Belgian TD children only, the GDA was positively linked to their ToM-Beliefs; in other words, the higher the cognitive abilities of these preschoolers, the better they understand beliefs and false beliefs. On the contrary, no significant link was obtained in Quebec TD preschoolers. In the Belgian group with ID, the older children were, the higher their ToM-Beliefs; their social adjustment and social skills were also positively and significantly linked to their CA and GDA, so older children who had a better cognitive level showed better-adjusted social behavior. In Quebec children with ID, their GDA was positively and significantly linked to their global ToM and their ToMI-Thoughts, ToMI-Beliefs, and ToMI-Socioemotional, but their CA was not significantly linked to any socio-emotional abilities; therefore, the higher these children's cognitive level, the better they understand cognitive mental states, thoughts, beliefs and false beliefs, and affective mental states.

In both cultural TD groups, the maternal educational level was linked to some abilities. Mothers' educational level was positively and significantly correlated with social adjustment and abilities to mobilize the ToM in a social context in Belgian TD children, and with affective ToM (socio-emotional) in Quebec TD children. So, the higher the educational level, the better these specific socioemotional abilities in TD preschoolers are in each cultural group.

Concerning significant predictors of ToM abilities in the entire sample, the children's GDA explained the variability of the global understanding of mental states and contributed to the variability of the understanding of thoughts, beliefs, 
and socio-emotional mental states. As shown in significant regression models: specifically, the combinations of GDA and other factors better predicted specific abilities in ToM. The socio-emotional ToM varies according to the higher educational level of mothers and children's GDA; the ToMI-Beliefs varies according to children's GDA, TD vs ID factor, the cultural group. Positive and predictive links between GDA and ToM abilities were also observed in previous studies, on TD children (Conte et al., 2019; Conte et al., 2018) and children with ID, e.g. (Baurain \& Nader-Grosbois, 2013a, 2013b; Charman \& Campbell, 2002; Jacobs, et al., 2020; Nader-Grosbois et al., 2013). The more children progress in their cognitive development, the more they develop their understanding of affective and cognitive mental states. More precisely, the understanding of the cognitive mental states of beliefs seems to be influenced by the presence or absence of ID in children, in the sense of greater difficulty in ID children than TD children, even with similar GDA, while this is not the case for affective ToM. These results tie in with those of most studies that observed a similar level in ToM emotions or affective ToM, between GDA-matched TD children and children with ID (Baurain \& Nader-Grosbois, 2013a, 2013b; Fiasse \& Nader-Grosbois, 2012; Garitte, 2003; Thirion-Marissiaux \& Nader-Grosbois, 2008b), but not with the observation of a lower level of affective ToM perceived by parents of children with ID than parents of TD children, by Légaré et al. (2019). As regards the difference in ToM-Beliefs depending on the TD or ID status, it is consistent with results that emphasized some deficits in the understanding of beliefs and false beliefs in cognitive ToM in children with ID (Baglio et al., 2016; Charman \& Campbell, 2002; Légaré et al., 2019; Thirion-Marissiaux \& Nader-Grosbois, 2008a).

Regarding global social adjustment in the entire sample, it was better predicted by the combination of the TD vs ID factor and the mothers' reprimand response to positive emotions. Particularly, the variability in social adjustment, by the mobilizing of ToM in daily life, was explained partially by the TD vs ID factor and by mothers' reprimand reaction to positive emotions; social skills relating to rules were better explained by the combination of TD vs ID factor, mothers' reprimand response to positive emotions, and encouragement and punition as responses to negative emotions, perceived by children. The variability in social adjustment and social skills depending on the TD vs ID status, despite a similar GDA in children, was in line with several results showing a lower level in this domain in children with ID, who also present a greater risk of externalizing or/and internalizing behavioral problems (Baker et al., 2003, 2011; Baurain \& Nader-Grosbois, 2013b; Hauser-Cram \& Woodman, 2016; Jacobs et al., 2020; Leffert \& Siperstein, 2002; Leffert, Siperstein, \& Millikan, 2000; Nader-Grosbois et al., 2013; Thirion-Marissiaux \& Nader-Grosbois, 2008c). The variability in social skills relating to rules, depending on the presence or absence of ID, maternal encouragement and punition in response to negative emotions, and reprimand in response to positive emotions could be explained by the fact that mothers of ID children displayed an openness to understanding their negative emotions, and tried to help them to better control the intensity of their emotional expres- 
sion, in accordance with rules in social contexts. Jacobs et al. (2019a) emphasized also that reprimand as a response to positive emotions in mothers of ID children was positively linked to some socially adjusted behavior by their children, who were notably cooperative, but another maternal supportive problemfocused reaction as a response to negative emotions was linked to greater confidence in the child.

Moreover, the variability of emotion regulation in children in the entire sample was partially explained by cultural group. However, it was the TD vs ID factor that contributed to variability in children's emotional dysregulation. These results are consistent with previous studies that showed, when the ID children are matched on developmental age with TD children, that no difference was observed in emotional regulation (Baurain \& Nader-Grosbois, 2012, 2013b), while children with ID have more emotional dysregulation than TD children (Baker et al., 2007; Berkovits, Eisenhower, \& Blacher, 2017). As regards the variability of emotional regulation partially due to cultural group, this ties in with the proposition of Jaramillo et al. (2017) concerning the impact of cultural context on self-regulation and emotion regulation in children. Other predictive links of individual factors (CA, GDA) and of specific reactions on the part of mothers or fathers were reported by Jacobs et al. (2019b) contributing to variability in emotional regulation or dysregulation in children with ID.

The first hypothesis about cross-cultural variability was partially confirmed for specific socio-emotional abilities in TD children only, in comparative analyses. The hypothesis was also partially confirmed because correlations between individual and cultural factors and socio-emotional abilities present different specific correlational patterns, in both In Belgian and Quebec groups, and significant regression models emphasized that socio-emotional abilities in children are predicted by either individual factors or maternal educational level and cultural factors, or a combination thereof, but in a variable way, for ToM, social adjustment, and emotional regulation or dysregulation. Observations about differences in TD children's emotion regulation and dysregulation as well as in social adjustment could be related to the preschool programs that they benefit from in Quebec and Belgium. Political decrees underline the different prioritization and implementation of socio-emotional activities in preschool classrooms in these two countries, compared with learning activities. As regards teachers' education, a difference could be identified in the area of evidence-based practices (Abdeljalil \& Changkakoti, 2009; Potvin et al., 2018). Parents' level of education and socialization behaviors could also have a variable impact on children's socio-emotional development (Claes et al., 2008; Parent et al., 2008; Roskam et al., 2015). Finally, different patterns of emotional experiences and of social rules could influence both the way the child expresses and regulates their emotions and the parental practices in socio-emotional domains (Gottman et al., 1996; Halberstadt et al., 2001; Hofstede, 2001; Keller, 2007; Mesquita \& Walker, 2003). 


\section{Comparison of Belgian and Quebec mothers reactions to emotions to-} ward their TD and ID children, and individual, educational, cultural factors

Secondly, the study explored similarities vs differences between Belgian and Quebec mothers who are French speaking, in their reactions to emotions, toward and with their TD children or children with ID presenting similar developmental age, and the predictors. The second hypothesis postulated that mothers' supportive vs unsupportive reactions and specific reactions to positive and negative emotions vary according to the children's characteristics (GDA, TD vs ID factor) and socio-emotional abilities, and depending on the culture (Belgian vs Quebec) and their own educational level. In other words, this hypothesis suggested that supportive vs unsupportive reactions and specific reactions to these children's emotions could differ between Belgian and Quebec mothers. Different predictors could influence these maternal reactions.

As regards cross-cultural variability, the comparative analyses of reactions between Belgian and Quebec mothers of children with and without ID emphasized nuanced results.

As regards supportive and unsupportive reactions to negative and positive emotions of the TD children, Quebec mothers did not react differently from Belgian mothers. However, a unique cultural difference is identified: mothers from Quebec displayed more socialization reactions when their child feels positive emotions than Belgian mothers. Toward ID children, there is a cross-cultural difference in unsupportive reactions to the child's negative emotions, with Belgian mothers reporting more unsupportive reactions overall, and specifically displaying more punitive and minimizing reactions than Quebec mothers. However, Belgian mothers reported more supportiveness by comforting their child who feels negative emotions than Quebec mothers, while Quebec mothers showed more encouragement of expression of negative emotions by their child than Belgian mothers. In addition, other supportive or unsupportive reactions to positive emotions did not differ between the two cultural groups of mothers of children with ID. Some cultural differences in mothers' reactions to negative emotions could be, perhaps, related to the type of parental support for determining which strategies of socialization and scaffolding by parents should be more protective $v s$ risk factors toward the child's socio-emotional development (Halberstadt \& Lozada, 2011) and related to partnership between professionals and parents, particularly with a low educational level (Abdeljalil \& Changkakoti, 2009). The quality of support given to parents by professionals in the French-speaking region in Belgium is increasingly being recognized by the Office de la Naissance et l'Enfance (ONE, 2012), which gave explicit guidelines to improve parental support. In Quebec, specific guidelines were given to adjust parenting to the child to stimulate his or her development, for example by the Centre Jeunesse de Montréal (Paquette, 2004).

Concerning predictors of maternal unsupportive reactions to negative emotions in the entire sample, they were partially explained by cultural group. Con- 
cerning maternal reactions to positive emotions, mothers' low educational level partially explained the variability of unsupportive reactions, while children's abilities in ToM in terms of Beliefs and Thoughts better explained variability in supportive reactions. Concerning factors explaining specific maternal reactions, the cultural factor partially explained mothers' minimization, distress, and encouragement in response to their children's negative emotions. The combination of cultural factor and children's GDA better explained variability in mothers' punitive reactions to their children's negative emotions. Mothers' low educational level contributed to the variability in their reprimand reactions to children's positive emotions. These results showed that the cultural factor has an impact on variability for specific unsupportive reactions and one supportive reaction by Belgian or Quebec mothers when the child with and without ID felt negative emotions. Moreover, the low educational level of mothers could be a risk factor for maternal use of unsupportive reactions, and particularly reprimand reactions when the child with or without ID felt positive emotions. These results regarding variability according to both socio-cultural aspects (the contrast between Belgian and Quebec mothers' reactions and variability in reported mothers' reactions, depending on the maternal educational levels) are consistent with the assumption about parent's socialization of emotions vary in quality or in type of practices depending on cultural frames, as proposed by several authors (Bornstein \& Bradley, 2003; Dunsmore \& Halberstadt, 1997, 2009; Halberstadt et al., 2001; Halberstadt \& Lozada, 2011; Trommsdorff \& Kornadt, 2003).

Another result that also emphasized the bidirectional links between parentchild behaviors was that better understanding of beliefs and thoughts in children with and without ID was positively linked to maternal supportive reactions; this is consistent with the bidirectionality concept postulated by Kuczynski et al. (1997).

The second hypothesis was confirmed on the basis of comparative analyses, correlations, and significant predictors obtained to explain mothers' reactions to children's positive and negative emotions.

\section{Limitations of this study}

Despite the interesting and nuanced results of this exploratory study, some limits should be specified. Firstly, the sample size was small in order to examine the respective impact of distinct individual factors and specific cultural factors on maternal reactions to emotions and children's profiles in ToM, emotion regulation and social adjustment. Secondly, the study used only indirect measures by means of questionnaires and not performance-based measures or observational design of socio-emotional abilities in children and of emotion-related reactions by mothers. Thirdly, this study is cross-sectional and concerns only a comparison between two French-speaking populations that limits the significance of findings. Fourthly, Quebec mothers of TD children and of ID children presented a higher educational level than Belgian mothers. These limitations could induce implications for future research. 


\section{Conclusion}

\section{Implications for future research}

First, although the interest of examining these questionings and hypotheses through the mothers' perceptions of their children's and their emotion-related reactions, it was also relevant to investigate them using performance-based measures such as the ToM Task Battery, or ToM emotions and ToM beliefs tasks, to children (as in studies led by (Jacobs et al., 2019a, 2019b; Mazzone \& NaderGrosbois, 2016, 2017a, 2017b, 2017c, 2017d)) and observation design to analyze maternal practices.

Secondly, assessment of executive functions and language abilities (beyond verbal and non-verbal developmental age) could be also relevant in order to determine cognitive and language influences in these links between socio-emotional abilities in children and reactions in parents.

Thirdly, longitudinal studies of TD children and of children with ID about their developmental trajectories in ToM, emotion regulation, and social adjustment, taking into account potential changes in reactions to emotions on the part of mothers and fathers, as well as individual and cultural factors, could help to better understand the dynamics of these processes.

Fourthly, the present study was focused only on reactions, but emotional discourse in family and parent-child conversations about emotions are also likely to support the child's socio-emotional development, as revealed by several studies on TD children and their parents, e.g. (Adrian et al., 2005; Lagattuta \& Wellman, 2002; Ornaghi et al., 2015; Ruffman et al., 2002; Ruffman et al., 2006; Taumoepeau \& Reese, 2013). However, very few studies investigated parent-child conversations about emotions involving children with ID, notably (Baker \& Crnic, 2009; Fenning, Baker, \& Juvonen, 2011; Jacobs et al., 2019a; Légaré et al., 2019; Tingley, Gleason, \& Hooshyar, 1994).

Finally, future research should examine links between emotion related conversations and reactions in parents of typically and atypically developing children, in different cultures, and their combined impacts on socio-emotional development (for example in parents of children with autism spectrum disorders in different countries, as the study led by (Nader-Grosbois et al., 2022)).

\section{Implications for intervention}

As regards implications for intervention, several programs have been developed to support parents in their emotional socialization role or as coaches of their child's emotions (for example, "Tuning into Kids" (Havighurst et al., 2010; Wilson, Havighurst, \& Harley, 2012)). Other evidence-based programs for children focused on training of their abilities in ToM or in social information processing, to help them to better understand mental states or activate steps of social problem-solving in critical social situations, to improve their emotion regulation or social adjustment and limit their risk of behavior problems, particularly externalizing disorders in children at preschool or school age (Honoré et al., 2020; Houssa, Nader-Grosbois, \& Jacobs, 2014; Houssa \& Nader-Grosbois, 
2016a, 2016b; Houssa, Jacobs, \& Nader-Grosbois, 2017; Houssa et al., 2017) and in ID children (Jacobs \& Nader-Grosbois, 2020a, 2020b). However, the implementation of these programs could be better encouraged. The guidelines and decrees making support for socio-emotional abilities in typically and atypically developing children in Belgium a priority, beyond learning and autonomy skills, appeared explicitly later than in Quebec, and also the reference to evidencebased programs to be implemented in school and in early intervention targeted children and parents. The follow-up of individual learning plans involving parents, teachers, and specialized professionals, which includes the implementation of socio-emotional evidence-based programs and periodic assessment of progression in children, could be better encouraged in Belgium and Quebec.

Although the efficacy of child-focused and/or parent-focused training programs was demonstrated to increase socio-emotional abilities in these children, it is important to consider not only specificities in socio-emotional profiles in children (Jacobs, Simon, \& Nader-Grosbois, 2020), but also parents' profiles, spontaneous ERSB practices, and cultural family factors, in prevention and intervention for children at developmental risk and their parents.

\section{Acknowledgements}

We would like to thank the Chair Baron Frère in special education and the Marguerite-Marie Delacroix Foundation for their financial support. We also are really grateful to all the children and parents who took part in our studies.

\section{Conflicts of Interest}

The authors declare no conflicts of interest regarding the publication of this paper.

\section{References}

Abbeduto, L., \& Murphy, M. M. (2004). Language, Social Cognition, Maladaptive Behavior, and Communication in Down Syndrome and Fragile X Syndrome. In M. L. Rice, \& S. F. Warren (Eds.), Developmental Language Disorders. From Phenotypes to Etiologies (pp. 77-97). Lawrence Erlbaum Associates Inc.

Abdeljalil, A., \& Changkakoti, N. (2009). Les relations entre parents et enseignants: Bilan des recherches récentes. La Revue internationale de l'éducation familiale, 25, 103-130. https://doi.org/10.3917/rief.025.0103

Adrian, J. E., Clemente, R. A., Villanueva, L., \& Rieffe, C. (2005). Parent-Child Picture Book Reading, Mothers' Mental State Language and Children's Theory of Mind. Journal of Child Language, 32, 673-686. https://doi.org/10.1017/S0305000905006963

American Association of Psychology (2013). Diagnostic and Statistical Manual of Mental Disorders (5th ed.). American Psychiatric Publishing.

Astington, J. W., \& Baird, J. A. (2005). Why Language Matters for Theory of Mind. Oxford University Press.https://doi.org/10.1093/acprof:oso/9780195159912.001.0001

Astington, J. W., \& Edward, M. J. (2010). The Development of Theory of Mind in Early Childhood. In R. G. Tremblay, R. Barr, V. Peters, \& M. Boivin (Eds.), Encyclopedia on Early Childhood Development (pp. 1-6). Centre of Excellence for Early Childhood Development. 
Astington, J. W., Repacholi, B., \& Slaughter, V. (2003). Individual Differences in Theory of Mind: Implications for Typical and Atypical Development. Psychology Press.

Baglio, G., Blasi, V., Sangiuliano Intra, F., Castelli, I., Massaro, D., Baglio, F., \& Marchetti, A. (2016). Social Competence in Children with Borderline Intellectual Functioning: Delayed Development of Theory of Mind across All Complexity Levels. Frontiers in Psychology, 7, Article No. 1604. https://doi.org/10.3389/fpsyg.2016.01604

Baker, B. L., McIntyre, L. L., Blacher, J., Crnic, K., Edelbrock, C., \& Low, C. (2003). Preschool Children with and without Developmental Delay: Behaviour Problems and $\mathrm{Pa}-$ renting Stress over Time. Journal of Intellectual Disability Research, 47, 217-230. https://doi.org/10.1046/j.1365-2788.2003.00484.x

Baker, J. K., \& Crnic, K. A. (2009). Thinking about Feelings: Emotion Focus in the Parenting of Children with Early Developmental Risk. Journal of Intellectual Disability Research, 53, 450-462. https://doi.org/10.1111/j.1365-2788.2009.01161.x

Baker, J. K., Fenning, R. M., Crnic, K. A., Baker, B. L., \& Blacher, J. (2007). Prediction of Social Skills in 6-Year-Old Children with and without Developmental Delays: Contributions of Early Regulation and Maternal Scaffolding. American Journal on Mental Retardation, 112, 375-391. https://doi.org/10.1352/0895-8017(2007)112[0375:POSSIY]2.0.CO;2

Baker, J. K., Smith, L. E., Greenberg, J. S., Seltzer, M. M., \& Taylor, J. L. (2011). Change in Maternal Criticism and Behavior Problems in Adolescents and Adults with Autism across a 7-Year Period. Journal of Abnormal Psychology, 120, 465-475.

https://doi.org/10.1037/a0021900

Baurain, C., \& Nader-Grosbois, N. (2012). Socio-Emotional Regulation in Children with Intellectual Disability and Typically Developing Children in Interactive Contexts. $E u-$ ropean Journal of Disability Research, 6, 75-93. https://doi.org/10.1016/j.alter.2012.02.001

Baurain, C., \& Nader-Grosbois, N. (2013a). Compétences sociales et émotionnelles: Enfant typique et déficient intellectuel. Presses AcadémiquesFrancophones.

Baurain, C., \& Nader-Grosbois, N. (2013b). Theory of Mind, Socio-Emotional ProblemSolving, Socio-Emotional Regulation in Children with Intellectual Disability and in Typically Developing Children. Journal of Autism and Developmental Disorders, 43, 1080-1097. https://doi.org/10.1007/s10803-012-1651-4

Bellugi, U., Lichtenberger, L., Jones, W., Lai, Z., \& St George, M. (2000). The Neurocognitive Profile of Williams Syndrome: A Complex Pattern of Strengths and Weaknesses. Journal of Cognitive Neuroscience, 12, 7-29. https://doi.org/10.1162/089892900561959

Berkovits, L., Eisenhower, A., \& Blacher, J. (2017). Emotion Regulation in Young Childrenwith Autism Spectrum Disorders. Journal of Autism and Developmental Disorders, 47, 68-79. https://doi.org/10.1007/s10803-016-2922-2

Bornstein, M., \& Bradley, R. (2003). Socioeconomic Status, Parenting, and Child Development. Lawrence Erlbaum Associates Inc.

Brown, G. L., Craig, A. B., \& Halberstadt, A. G. (2015). Parent Gender Differences in Emotion Socialization Behaviors Vary by Ethnicity and Child Gender. Parenting: Science and Practice, 15, 135-157. https://doi.org/10.1080/15295192.2015.1053312

Camras, L., Kolmodin, K., \& Chen, Y. (2008). Mothers' Self-Reported Emotional Expression in Mainland Chinese, Chinese American and European American Families. International Journal of Behavioral Development, 32, 459-463. https://doi.org/10.1177/0165025408093665

Charman, T., \& Campbell, A. (2002). Theory of Mind and Social Competence in Individ- 
uals with a Mental Handicap. Journal of Developmental and Physical Disabilities, 14, 263-276. https://doi.org/10.1023/A:1016076405731

Claes, M., Ziba-Tanguay, K., \& Benoit, A. (2008). La parentalité: Le role de la culture. In C. Parent, S. Drapeau, M. Brousseau, \& E. Pouliot, E. (Eds.), Visages multiples de la parentalité (pp. 3-31). Presses de l’Université du Québec.

Cole, P. M., \& Tan, P. Z. (2007). Emotion Socialization from a Cultural Perspective. In J. E. Grusec, \& P. D. Hastings (Eds.), Handbook of Socialization: Theory and Research (pp. 516-542). Guilford.

Comte-Gervais, I., Giron, A., Soares-Boucaud, I., \& Poussin, G. (2008). Evaluation de l'intelligence sociale chez l'enfant. L'information Psychiatrique, 84, 667-673. https://doi.org/10.3917/inpsy.8407.0667

Conte, E., Ornaghi, V., Grazzani, I., Pepe, A., \& Cavioni, V. (2019). Emotion Knowledge, Theory of Mind, and Language in Young Children: Testing a Comprehensive Conceptual Model. Frontiers in Psychology, 10, Article No. 2144. https://doi.org/10.3389/fpsyg.2019.02144

Coutu, S., Dubeau, D., Provost, M., Royer, N., \& Lavigueur, S. (2002). Validation de la version française du questionnaire: Coping with Children'sNegative Emotions ScaleCCNES. Canadian Journal of Behavioural Science, 34, 230-234. https://doi.org/10.1037/h0087175

Daffe, V., \& Nader-Grosbois, N. (2009). Réactions parentales face aux émotions de leur enfant: Adaptation intégrée de deux instruments. In N. Nader-Grosbois (Ed.), Résilience, régulation et qualité de vie: Concepts, évaluation et intervention (pp. 143-159). Presses Universitaires de Louvain.

Degnan, K., Almas, A. N., \& Fox, N. A. (2010). Temperament and the Environment in the Etiology of Childhood Anxiety. Journal of Child Psychology and Psychiatry, 51, 497-517. https://doi.org/10.1111/j.1469-7610.2010.02228.x

Deneault, J., \& Ricard, M. (2013). Are Emotion and Mind Understanding Differently Linked to Young Children's Social Adjustment? Relationships between Behavioral Consequences of Emotions, False Belief, and SCBE. The Journal of Genetic Psychology, 174, 88-116. https://doi.org/10.1080/00221325.2011.642028

Denham, S. A. (1998). Emotional Development in Young Children. Guilford Press.

Denham, S. A., \& Kochanoff, A. (2002). “Why is she crying?”: Children's Understanding of Emotion from Preschool to Preadolescence. In L. F. Barrett, \& P. Salovey (Eds.), The Wisdom in Feeling: Psychological Processes in Emotional Intelligence (pp. 239-270). Guilford Press.

Denham, S. A., \& Weissberg, R. P. (2004). Social-Emotional Learning in Early Childhood: What We Know and Where to Go from Here. In E. Chesebrough, P. King, T. P. Gullotta, \& M. Bloom (Eds.), A Blueprint for the Promotion of Prosocial Behavior in Early Childhood (pp. 13-50). Kluwer/Academic.

Denham, S. A., Bassett, H. H., \& Wyatt, T. (2007). The Socialization of Emotional Competence. In J. E. Grusec, \& P. D. Hastings (Eds.), Handbook of Socialization: Theory and Research (pp. 614-637). Guilford Press.

Denham, S. A., Blair, K. A., DeMulder, E., Levitas, J., Sawyer, K. S., Auerbach-Major, S. T. et al. (2003). Preschoolers' Emotional Competence: Pathway to Mental Health? Child Development, 74, 238-256. https://doi.org/10.1111/1467-8624.00533

Denham, S. A., Caal, S., Bassett, H. H., Benga, O., \& Geangu, E. (2004). Listening to Parents: Cultural Variations in the Meaning of Emotions and Emotion Socialization. Cognitie, Creier, Comportament, 8, 321-350. 
Denham, S. A., Mitchell-Copeland, J., Strandberg, K., Auerbach, S., \& Blair, K. (1997). Parental Contributions to Preschoolers' Emotional Competence: Direct and Indirect Effects. Motivation and Emotion, 27, 65-86.

https://doi.org/10.1023/A:1024426431247

Denham, S. A., Zoller, D., \& Couchoud, E. A. (1994). Socialization of Preschoolers' Emotion Understanding. Developmental Psychology, 30, 928-936.

https://doi.org/10.1037/0012-1649.30.6.928

Dodge, K. A., \& Pettit, G. S. (2003). A Biopsychosocial Model of the Development of Chronic Conduct Problems in Adolescence. Developmental Psychology, 39, 349-371.

https://doi.org/10.1037/0012-1649.39.2.349

Dunsmore, J. C., \& Halberstadt, A. G. (1997). How Does Family Emotional Expressiveness Affect Children's Schemas? New Directions for Child Development, 77, 45-66. https://doi.org/10.1002/cd.23219977704

Dunsmore, J. C., \& Halberstadt, A. G. (2009). The Dynamic Context of Children's Emotions: Family and Cultural System Influences. In J. A. Mancini, \& K. A. Roberto (Eds.), Pathways of Human Development: Explorations of Change (pp. 171-190). Lexington Books.

Duyme, M., \& Capron, C. (2010). L’Inventaire du Développement de l'Enfant (IDE). Normes et validation françaises du Child Development Inventory (CDI). Devenir, 22, 13-26. https://doi.org/10.3917/dev.101.0013

Duyme, M., Capron, C., \& Zorman, M. (2010). L’Inventaire du Développement de l'Enfant (IDE): Manuel d'utilisation. Devenir, 1, 27-50.

https://doi.org/10.3917/dev.101.0027

Eisenberg, N., Cumberland, A., \& Spinrad, T. L. (1998a). Parental Socialization of Emotion. Psychological Inquiry, 9, 241-273. https://doi.org/10.1207/s15327965pli0904_1

Eisenberg, N., Fabes, R. A., \& Murphy, B. C. (1996). Parents' Reactions to Children's Negative Emotions: Relations to Children's Social Competence and Comforting Behavior. Child Development, 67, 2227-2247. https://doi.org/10.2307/1131620

Eisenberg, N., Gershoff, E. T., Fabes, R. A., Shepard, S. A., Cumberland, A., Losoya, S. et al. (2001a). Mothers' Emotional Expressivity and Children's Behavior Problems and Social Competence: Mediation through Children's Regulation. Developmental Psychology, 37, 475-490. https://doi.org/10.1037/0012-1649.37.4.475

Eisenberg, N., Losoya, S., Fabes, R. A., Guthrie, I. K., Reiser, M., Murphy, B. et al. (2001b). Parental Socialization of Children's Dysregulated Expression of Emotion and Externalizing Problems. Journal of Family Psychology, 15, 183-205.

https://doi.org/10.1037/0893-3200.15.2.183

Eisenberg, N., Spinrad, T. L., \& Cumberland, A. (1998b). The Socialization of Emotion: Reply to Commentaries. PsychologicalInquiry, 9, 317-333.

https://doi.org/10.1207/s15327965pli0904_17

Eisenberg, N., Zhou, Q., Losoya, S. H., Fabes, R. A., Shepard, S. A., Murphy, B. C. et al. (2003). The Relations of Parenting, Effortful Control, and Ego Control to Children's Emotional Expressivity. Child Development, 74, 875-895.

https://doi.org/10.1111/1467-8624.00573

Fabes, R. A., Leonard, S. A., Kupanoff, K., \& Martin, C. L. (2001). Parental Coping with Children's Negative Emotions: Relations with Children's Emotional and Social Responding. Child Development, 72, 907-920. https://doi.org/10.1111/1467-8624.00323

Fabes, R. A., Poulin, R. E., Eisenberg, N., \& Madden-Derdich, D. A. (2002). The Coping with Children's Negative Emotions Scale (CCNES): Psychometric Properties and Rela- 
tions with Children's Emotional Competence. In R. A. Fabes (Ed.), Emotions and the Family (pp. 285-309). The Haworth Press, Inc.

Fenning, R. M., Baker, B. L., \& Juvonen, J. (2011). Emotion Discourse, Social Cognition, and Social Skills in Children with and without Developmental Delays. Child Development, 82, 717-731. https://doi.org/10.1111/j.1467-8624.2010.01569.x

Fiasse, C., \& Nader-Grosbois, N. (2012). Perceived Social Acceptance, Theory of Mind and Social Adjustment in Children with Intellectual Disabilities. Research in Developmental Disabilities, 33, 1871-1880. https://doi.org/10.1016/j.ridd.2012.05.017

Fivush, R., \& Wang, Q. (2005). Emotion Talk in Mother-Child Conversations of the Shared Past: The Effects of Culture, Gender, and Event Valence. Journal of Cognition and Development, 6, 489-506. https://doi.org/10.1207/s15327647jcd0604_3

Flavell, J. H. (1999). Cognitive Development: Children's Knowledge about the Mind. Annual Review of Psychology, 50, 21-45. https://doi.org/10.1146/annurev.psych.50.1.21

Garner, P. W. (2006). Prediction of Prosocial and Emotional Competence from Maternal Behavior in African American Preschoolers. Cultural Diversity \& Ethnic Minority Psychology, 12, 179-198. https://doi.org/10.1037/1099-9809.12.2.179

Garner, P. W., \& Estep, K. M. (2001). Emotional Competence, Emotion Socialization, and Young Children's Peer-Related Social Competence. Early Education and Development, 12, 29-48. https://doi.org/10.1207/s15566935eed1201_3

Garner, P. W., \& Spears, F. M. (2000). Emotion Regulation in Low-Income Preschoolers. Social Development, 9, 246-264. https://doi.org/10.1111/1467-9507.00122

Garner, P. W., Dunsmore, J. C., \& Southam-Gerrow, M. (2008). Mother-Child Conversations about Emotions: Linkages to Child Aggression and Prosocial Behavior. Social Development, 17, 259-277. https://doi.org/10.1111/j.1467-9507.2007.00424.x

Garner, P. W., Jones, D. C., \& Miner, J. L. (1994). Social Competence among Low-Income Preschoolers: Emotion Socialization Practices and Social Cognitive Correlates. Child Development, 65, 622-637. https://doi.org/10.2307/1131405

Garner, P. W., Jones, D. C., Gaddy, G., \& Rennie, K. (1997a). Low-Income Mothers' Conversations about Emotions and Their Children's Emotional Competence. Social Development, 6, 37-52. https://doi.org/10.1111/j.1467-9507.1997.tb00093.x

Garner, P. W., Robertson, S., \& Smith, G. (1997b). Preschool Children's Emotional Expressions with Peers: The Roles of Gender and Emotion Socialization. Sex Roles, 36, 675-691. https://doi.org/10.1023/A:1025601104859

Gottman, J. M., Katz, L. F., \& Hooven, C. (1996). Parental Meta-Emotion Philosophy and Theemotional Life of Families: Theoretical Models and Preliminary Data. Journal of Family Psychology, 10, 243-268. https://dx.doi.org/10.1037/0893-3200.10.3.243

Grusec, J. E. (2002). Parental Socialization and Children's Acquisition of Values. In M. H. Bornstein (Ed.), Handbook of Parenting (2nd ed., Vol. 5, pp. 143-167). Lawrence Erlbaum Associates Inc.

Halberstadt, A. G., \& Lozada, F. T. (2011). Emotion Development in Infancy through the Lens of Culture. Emotion Review, 3, 158-168. https://doi.org/10.1177/1754073910387946

Halberstadt, A. G., Denham, S. A., \& Dunsmore, J. (2001). Affective Social Competence. Social Development, 10, 79-119. https://doi.org/10.1111/1467-9507.00150

Hauser-Cram, P., \& Woodman, A. C. (2016). Trajectories of Internalizing and Externalizing Behavior Problems in Children with Developmental Disabilities. Journal of $A b$ normal Child Psychology, 44, 811-821. https://doi.org/10.1007/s10802-015-0055-2

Havighurst, S. S., Wilson, K. R., Harley, A. E., Prior, M. R., \& Kehoe, C. (2010). Tuning in 
to Kids: Improving Emotion Socialization Practices in Parents of Preschool Children Findings from a Community Trial. Journal of Child Psychology and Psychiatry, 51, 1342-1350. https://doi.org/10.1111/j.1469-7610.2010.02303.x

Hoffman, M. L. (2000). Empathy and Moral Development. Cambridge University Press. https://doi.org/10.1017/CBO9780511805851

Hofstede, G. (2001). Culture's Consequences: Comparing Values, Behaviors, Institutions and Organizations across Nations. Sage Publications.

Honoré, N., Houssa, M., Volckaert, A., Noël, M.-P., \& Nader-Grosbois, N. (2020). Training Inhibition and Social Cognition in the Classrooms. Frontiers in Psychology, 11, Article No. 1974. https://doi.org/10.3389/fpsyg.2020.01974

Houssa, M., \& Nader-Grosbois, N. (2016a). Could Social Cognition Training Reduce Externalizing Behaviors and Social Maladjustment in Preschoolers? Journal of Psychological Abnormalities, S1, 1-10. https://doi.org/10.4172/2471-9900.S1-005

Houssa, M., \& Nader-Grosbois, N. (2016b). Experimental Study of Middle-Term Training in Social Cognition in Preschoolers. Journal of Education and Training Studies, 4, 61-73. https://doi.org/10.11114/jets.v4i1.972

Houssa, M., Jacobs, E., \& Nader-Grosbois, N. (2017). Impact of Short-Term Training in Social Cognition in Preschoolers with Externalizing Behavior. Journal of Education and Training Studies, 5, 110-123. https://doi.org/10.11114/jets.v5i1.2081

Houssa, M., Mazzone, S., \& Nader-Grosbois, N. (2014). Validation d'une version francophone de l'inventaire de la Théorie de l'Esprit (ToMI-vf). Revue Européenne de Psychologie Appliquée, 64, 169-179. https://doi.org/10.1016/j.erap.2014.02.002

Houssa, M., Nader-Grosbois, N., \& Jacobs, E. (2014). Experimental Study of Short-Term Training in Social Cognition in Pre-Schoolers. Journal of Education and Training Studies, 2, 139-154. https://doi.org/10.11114/jets.v2i1.181

Houssa, M., Volckaert, A., Nader-Grosbois, N., \& Noël, M.-P. (2017). Differential Impact of an Executive-Function and a Social Cognition Training on Preschoolers with Externalizing Behavior Problems. Journal of Behavioral and Brain Science, 7, 598-620. https://doi.org/10.4236/jbbs.2017.712042

Howes, C. (2000). Social-Emotional Classroom Climate in Child Care, Child-Teacher Relationships and Children's Second Grade Peer Relations. Social Development, 9, 191-204. https://doi.org/10.1111/1467-9507.00119

Hudson, J. L., \& Rapee, R. M. (2001). Parent-Child Interactions and Anxiety Disorders: An Observational Study. Behaviour Research and Therapy, 39, 1411-1427. https://doi.org/10.1016/S0005-7967(00)00107-8

Hughes, C., Soares-Boucaud, I., Hochmann, J., \& Frith, U. (1997). Social Behaviour Inpervasive Developmental Disorders: Effects of Informant, Group and "Theory-of-Mind". European Child \& Adolescent Psychiatry, 6, 191-198. https://doi.org/10.1007/BF00539925

Hurrell, K., Hudson, J. L., \& Schniering, C.A. (2015). Parental Reactions to Children's Negative Emotions: Relationships with Emotion Regulation in Children with an Anxiety Disorder. Journal of Anxiety Disorders, 29, 72-82. https://doi.org/10.1016/j.janxdis.2014.10.008

Hutchins, T. L., Prelock, P. A., \& Bonazinga, L. (2012). Psychometric Evaluation of the Theory of Mind Inventory (ToMI): A Study of Typically Developing Children and Children with Autism Spectrum Disorder. Journal of Autism and Developmental Disorders, 42, 327-341. https://doi.org/10.1007/s10803-011-1244-7

Ireton, H. (1992). Child Development Inventories Manual. Behavior Science Systems. 
https://doi.org/10.1037/t05317-000

Ireton, H., \& Glascoe, F. P. (1995). Assessing Children's Development Using Parents' Reports: The Child Development Inventory. Clinical Pediatrics, 34, 248-255. https://doi.org/10.1177/000992289503400504

Jacobs, E., \& Nader-Grosbois, N. (2020a). Affective and Cognitive Theory of Mind in Children with Intellectual Disabilities: How to Train Them to Foster Social Adjustment and Emotion Regulation? Journal of Education and Training Studies, 8, 80-97. https://doi.org/10.11114/jets.v8i4.4757

Jacobs, E., \& Nader-Grosbois, N. (2020b). Training Social Information Processing in Elementary School Children with Intellectual Disabilities: A Key to Support Their Emotion Regulation and Social Behaviors. Journal of Education and Training Studies, 8, 7-22. https://doi.org/10.11114/jets.v8i6.4840

Jacobs, E., Mazzone, S., Simon, P., \& Nader-Grosbois, N. (2019a). The Unforeseen Influence of Parents' Socialization Behaviors on the Social Adjustment of Children with Intellectual Disabilities. Psychology, 10, 1275-1301.

https://doi.org/10.4236/psych.2019.109083

Jacobs, E., Mazzone, S., Simon, P., \& Nader-Grosbois, N. (2019b). The Unexpected Impact of Parental Emotional Socialization on Theory of Mind and Emotion Regulation: The Case of Children with Intellectual Disabilities. Psychology, 10, 1302-1332. https://doi.org/10.4236/psych.2019.109084

Jacobs, E., Simon, P., \& Nader-Grosbois, N. (2020). Social Cognition in Children with Non-Specific Intellectual Disability: An Exploratory Study. Frontiers in Psychology, 11, Article No. 1884. https://doi.org/10.3389/fpsyg.2020.01884

Jaramillo, J.M., Rendón, M.I., Muñoz, L., Weis, M., \& Trommsdorff, G. (2017). Children's Self-Regulation in Cultural Contexts: The Role of Parental Socialization Theories, Goals, and Practices. Frontiers in Psychology, 8, Article No. 923.

https://doi.org/10.3389/fpsyg.2017.00923

Jones, S., Eisenberg, N., Fabes, R. A., \& MacKinnon, D. P. (2002). Parents' Reactions to Elementary School Children's Negative Emotions: Relations to Social and Emotional Functioning at School. Merrill-Palmer Quarterly, 48, 133-159.

https://doi.org/10.1353/mpq.2002.0007

Keller, H. (2007). Cultures of Infancy. Lawrence Erlbaum Associates Inc.

Kuczynski, L., Marshall, S., \& Schell, K. (1997). Value Socialization in a Bidirectional Context. In J. E. Grusec,\& L. Kuczynski (Eds.), Parenting and Children's Internalization of Values: A Handbook of Contemporary Theory(pp. 23-50). Wiley.

Ladouceur, C., Reid, L., \& Jacques, A. (2002). Construction et validation du Questionnaire sur les réactions parentales aux émotions positives exprimées par l'enfant. Canadian. Journal of Behavioural Science, 34, 8-18. https://doi.org/10.1037/h0087150

Lagattuta, K. H., \& Wellman, H. M. (2002). Differences in Early Parent-Child Conversations about Negative versus Positive Emotions: Implications for the Development of Psychological Understanding. Developmental Psychology, 38, 564-580. https://doi.org/10.1037/0012-1649.38.4.564

Leffert, J. S., \& Siperstein, G. N. (2002). Social Cognition: A Key to Understanding Adaptive Behavior in Individuals with Mild Mental Retardation. International Review of Research in Mental Retardation, 25, 135-181. https://doi.org/10.1016/S0074-7750(02)80008-8

Leffert, J. S., Siperstein, G. N., \& Millikan, E. (2000). Understanding Social Adaptation in Children with Mental Retardation: A Social-Cognitive Perspective. Exceptional Children, 66, 530-545. https://doi.org/10.1177/001440290006600406 
Légaré, C., Morin, D., Poirier, N., \& Nader-Grosbois, N. (2019). Parental Socialization of Emotion: Differences in Mothers of Children with and without Intellectual Disability. Psychology, 10, 2062-2079. https://doi.org/10.4236/psych.2019.1015133

Mazzone, S., \& Nader-Grosbois, N. (2015). Comment les parents socialisent-ils les émotions de leur enfant? In M. Mikolajczak,\& I. Roskam (Eds.), Stress et défis de la parentalité (pp. 153-168). De Boeck.

Mazzone, S., \& Nader-Grosbois, N. (2016). How Are Parental Reactions to Children's Emotions Related to Their Theory of Mind Abilities? Psychology, 7, 166-179. https://doi.org/10.4236/psych.2016.72019

Mazzone, S., \& Nader-Grosbois, N. (2017a). Emotion-Related Socialization Behavioursin Parents of Children with an Autism Spectrum Disorder. Psychology, 8, 1134-1160. https://doi.org/10.4236/psych.2017.88074

Mazzone, S., \& Nader-Grosbois, N. (2017b). How Are Parental Reactions to Children's Emotions Linked with Theory of Mind in Children with Autism Spectrum Disorder? Research in Autism Spectrum Disorders, 40, 41-53. https://doi.org/10.1016/j.rasd.2017.05.003

Mazzone, S., \& Nader-Grosbois, N. (2017c). Variability and Predictors of Mothers and Fathers' Socialization Behaviors and Bidirectional Links with Their Preschoolers SocioEmotional Competences. Journal of Behavioral and Brain Science, 7, 621-653. https://doi.org/10.4236/jbbs.2017.712043

Mazzone, S., Roskam, I., Mikolajczak, M., \& Nader-Grosbois, N. (2017d). Do Parents Talk about Emotions with Their Children? The Questionnaire of Parent-Child Conversations about Emotions (QPCCE). Psychology, 8, 987-1007.

https://doi.org/10.4236/psych.2017.87065

Mervis, C. B., \& Velleman, S. L. (2011). Children with Williams Syndrome: Language, Cognitive, and Behavioral Characteristics and Their Implications for Intervention. Perspectives on Language Learning and Education, 18, 98-107. https://doi.org/10.1044/lle18.3.98

Mesquita, B., \& Walker, R. (2003). Cultural Differences in Emotions: A Context for Interpreting Emotional Experiences. Behaviour Research and Therapy, 41, 777-793. https://doi.org/10.1016/S0005-7967(02)00189-4

Morris, A. S., Silk, J. S., Steinberg, L., Myers, S. S., \& Robinson, L. R. (2007). The Role of the Family Context in the Development of Emotion Regulation. Social Development, 16, 361-388. https://doi.org/10.1111/j.1467-9507.2007.00389.x

Nader-Grosbois, N. (2011). La théorie de l'esprit: Entre cognition, émotion et adaptation sociale. De Boeck. https://doi.org/10.3917/dbu.nader.2011.01

Nader-Grosbois, N., \& Mazzone, S. (2014). Emotion Regulation, Personality and Social Adjustment in Children with Autism Spectrum Disorders. Psychology, 5, 1750-1767. https://doi.org/10.4236/psych.2014.515182

Nader-Grosbois, N., Houssa, M., \& Mazzone, S. (2013). How Could Theory of Mind Contribute to the Differentiation of Social Adjustment Profiles of Children with Externalizing Behavior Disorders and Children with Intellectual Disabilities? Research in Developmental Disabilities, 34, 2642-2660. https://doi.org/10.1016/j.ridd.2013.05.010

Nader-Grosbois, N., Jacobs, E., Mazzone, S., \& Poirier, N. (2022). Mothers' Socialization of Emotions and Theory of Mind and Emotion Regulation in Children with Autism Spectrum Disorders. Psychology, Speciallssue "Family Psychology".

Nédélec-Trohel, I., Numa-Bocage, L., \& Kalubi, J.-C. (2015). Conceptions, pratiques et formations inclusives dans l'espace francophone (France, Suisse, Belgique, Québec). La 
nouvelle revue de l'adaptation et de la scolarisation, 70, 19-23.

https://doi.org/10.3917/nras.070.0019

OECD (2018). Supporting Quality Early Childhood Education and Care through Workforce Development and Working Conditions. In Engaging Young Children: Lessons from Research about Quality in Early Childhood Education and Care (pp. 65-94). OECD Publishing. https://doi.org/10.1787/9789264085145-6-en

ONE (2012). Pour un accompagnement réfléchi des parents: un référentiel de soutien à la parentalité. Bruxelles. https://www.one.be/fileadmin/user_upload/siteone/PRO/SOUPA/Referentiel_soutien a_la_parentalite_ONE.pdf

Ornaghi, V., Grazzani, I., Cherubin, E., Conte, E., \& Piralli, F. (2015). "Let's Talk about Emotions!”. The Effect of Conversational Training on Preschoolers' Emotion Comprehension and Prosocial Orientation. Social Development, 24, 166-183.

https://doi.org/10.1111/sode.12091

Paczkowski, E., \& Baker, B. L. (2007). Parenting Children with and without Developmental Delay: The Role of Self-Mastery. Journal of Intellectual Disability Research, 51, 435-446. https://doi.org/10.1111/j.1365-2788.2006.00894.x

Paquette, F. (2004). A chaque enfant son projet de vie permanent. Centre jeunesse de Montréal, Institut Universitaire.

Parent, C., Drapeau, S., Brousseau, M., \& Pouliot, E. (2008). Visages multiples de la parentalité. Presses de l'Université du Québec.

Potvin, M., Dhume, F., Verhoeven, M., \& Ogay, T. (2018). La formation des enseignants sur la diversité et les rapports ethniques: Regard comparative France, Québec, Belgique et Suisse. Education et Francophonie, 46, 30-50. https://doi.org/10.7202/1055560ar

Rodas, N. V., Chavira, D. A., \& Baker, B. L. (2017). Emotion Socialization and Internalizing Behavior Problems in Diverse Youth: A Bidirectional Relationship across Childhood. Research in Developmental Disabilities, 62, 15-25.

https://doi.org/10.1016/j.ridd.2017.01.010

Rodas, N. V., Zeedyk, S. M., \& Baker, B. L. (2016). Unsupportive Parenting and InternalisingBehaviour Problems in Children with or without Intellectual Disability. Journal of Intellectual Disability Research, 60, 1200-1211.https://doi.org/10.1111/jir.12332

Roskam, I., Galdiolo, S., Meunier, J.-C., \& Stiévenart, M. (2015). Psychologie de la parentalité. De Boeck.

Ruffman, T., Slade, L., \& Crowe, E. (2002). The Relation between Children's and Mothers' Mental State Language and Theory of Mind Understanding. Child Development, 73, 734-751. https://doi/org/10.1111/1467-8624.00435

Ruffman, T., Slade, L., Devitt, K., \& Crowe, E. (2006). What Mothers Say and What They Do: The Relation between Parenting, Theory of Mind, Language and Conflict/Cooperation. British Journal of Developmental Psychology, 24, 105-124. https://doi.org/10.1348/026151005X82848

Saarni, C. (1999). The Development of Emotional Competence. Guilford Press.

Schalock, R., Borthwick-Duffy, S., Bradley, V., Buntinx, W., Coulter, D., Craig, E. et al. (2010). Intellectual Disability. Definition, Classification, and Systems of Support (11th ed.). American Association on Intellectual and Developmental Disabilities.

Shields, A., \& Cicchetti, D. (1997). Emotion Regulation among School Age Children: The Development and Validation of a New Criterion Q-Sort Scale. Developmental Psychology, 33, 906-916. https://doi.org/10.1037/0012-1649.33.6.906

Taumoepeau, M., \& Ruffman, T. (2006). Mother and Infant Talk About Mental States Re- 
lates to Desire Language and Emotion Understanding. Child Development, 77, 465-481. https://doi.org/10.1111/j.1467-8624.2006.00882.x

Thirion-Marissiaux, A.-F., \& Nader-Grosbois, N. (2008a). Theory of Mind "Beliefs", Developmental Characteristics and Social Understanding in Children and Adolescents with Intellectual Disabilities. Research in Developmental Disabilities, 29, 547-566. https://doi.org/10.1016/j.ridd.2007.09.004

Thirion-Marissiaux, A.-F., \& Nader-Grosbois, N. (2008b). Theory of Mind "Emotion", Developmental Characteristics and Social Understanding in Children and Adolescents with Intellectual Disabilities. Research in Developmental Disabilities, 29, 414-430. https://doi.org/10.1016/j.ridd.2007.07.001

Thirion-Marissiaux, A.-F., \& Nader-Grosbois, N. (2008c). Theory of Mind and SocioAffective Abilities in Disabled Children and Adolescents. European Journal of Disability Research, 2, 133-155. https://doi.org/10.1016/j.alter.2008.02.003

Tingley, E. C., Gleason, J. B., \& Hooshyar, N. T. (1994). Mothers' Lexicon of Internal State Words in Speech to Children with Down Syndrome and to Nonhandicapped Children at Mealtime. Journal of communication disorders, 27, 135-155.

Trommsdorff, G., \& Kornadt, H. J. (2003). Parent-Child Relations in Cross-Cultural Perspective. In L. Kuczynski (Ed.), Handbook of Dynamics in Parent-Child Relations (pp. 271-306). Sage. https://doi.org/10.4135/9781452229645.n14

Vygotsky, L. (1978). Interaction between Learning and Development. Readings on the Development of Children, 23, 34-41.

Wang, Q. (2001). “Did You Have Fun?” American and Chinese Mother-Child Conversations about Shared Emotional Experiences. Cognitive Development, 16, 693-715. https://doi.org/10.1016/S0885-2014(01)00055-7

Wechsler, D. (2004). Echelle d'intelligence de Wechsler pour la période préscolaire et primaire (Vol. 3). Éditions du Centre de Psychologie Appliquée.

Wilson, K. R., Havighurst, S. S., \& Harley, A. E. (2012). Tuning in to Kids: An Effectiveness Trial of a Parenting Program Targeting Emotion Socialization of Preschoolers. Journal of Family Psychology, 26, 56-65.https://doi.org/10.1037/a0026480

Wong, M. S., Diener, M. L., \& Isabella, R. A. (2008). Parents' Emotion Related Beliefs and Behaviorsand Child Grade: Associations with Children's Perceptions of Peer Competence. Journal of Applied Developmental Psychology, 29, 175-186. https://doi.org/10.1016/j.appdev.2008.02.003

Wong, M. S., McElwain, N. L., \& Halberstadt, A. G. (2009). Parent, Family, and Child Characteristics: Associations with Mother- and Father-Reported Emotion Socialization Practices. Journal of Family Psychology, 23, 452-463. https://doi.org/10.1037/a0015552

Yeates, K. O., Bigler, E. D., Dennis, M., Gerhardt, C. A., Rubin, K. H., Stancin, T., \& Vannatta, K. (2007). Social Outcomes in Childhood Brain Disorder: A Heuristic Integration of Social Neuroscience and Developmental Psychology. Psychological Bulletin, 133, 535-556. https://doi.org/10.1037/0033-2909.133.3.535 


\section{Appendix}

Appendix A. Example of one item of the Parental reactions questionnaire describing a situation eliciting negative emotion.

If my child lost an object that is important to him/her (his/her blanket) and reacts by crying:

a. I'm angry with him/her for not paying attention and then crying about it.

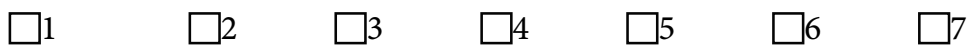

b. I tell my child that his or her reaction is exaggerated.

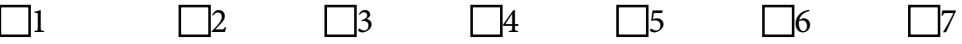

c. I help my child to think about places he/she hasn't looked before.
$\square 1$

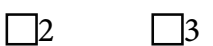
$\square 4 \quad \square 5$
$\square 6$

d. I distract my child by talking about happy things.

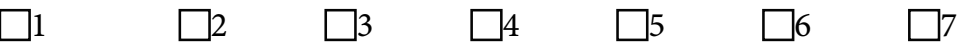

e. I tell him/her that it is normal to cry when you are sad.

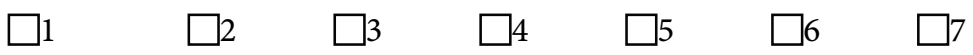

Appendix B. Example of one item of the Parental reactions questionnaire describing a situation eliciting positive emotion.

If we are at a wedding ceremony in the church and my child is laughing with his/her little cousin sitting behind him/her:

a. I smile at my child to indicate my understanding.

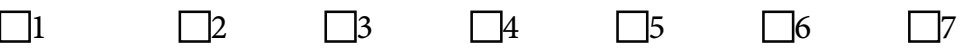

b. I tell my child to turn around and be quiet.

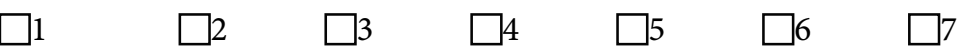

c. I feel embarrassed by my child's behavior.

$\square 1 \quad \square 2 \quad \square 3 \quad \square 4 \quad \square 5 \quad \square 6 \quad \square 7$

d. I tell my child not to laugh during a wedding ceremony because it upsets people things.

$\square 1 \quad \square 2 \quad \square 3 \quad \square 4 \quad \square 5 \quad \square 6 \quad \square 7$ 